Euskal ikerketen aldizkaria | Revue d'études basques |

Revista de estudios vascos | Basque studies review

$11 \mid 2006$

Numéro XI

\title{
Le gouvernement local dans une commune basque sous le Second Empire. Présentation du manuscrit Berrouet
}

\section{Xabier Itçaina}

\section{OpenEdition}

\section{Journals}

Édition électronique

URL : http://journals.openedition.org/lapurdum/342

DOI : 10.4000/lapurdum.342

ISSN : 1965-0655

Éditeur

IKER

Édition imprimée

Date de publication : 1 novembre 2006

Pagination : 203-240

ISBN : 978-2-86781-474-7

ISSN : $1273-3830$

Référence électronique

Xabier Itçaina, « Le gouvernement local dans une commune basque sous le Second Empire.

Présentation du manuscrit Berrouet », Lapurdum [En ligne], 11 | 2006, mis en ligne le 01 avril 2008,

consulté le 01 mai 2019. URL : http://journals.openedition.org/lapurdum/342 ; DOI : 10.4000/

lapurdum.342 


\title{
Le gouvernement local dans une commune basque sous le Second Empire. Présentation du manuscrit Berrouet
}

\author{
Xabier ITÇAINA \\ CNRS-SPIRIT, Science Politique Relations Internationales Territoire \\ Sciences Po Bordeaux \\ x.itcaina@sciencespobordeaux.fr
}

$\mathrm{La}^{1}$ publication des mémoires manuscrites de Guillaume Berrouet, maire d'Itxassou entre 1860 et 1870 se justifie à plusieurs égards. Ce texte, signé en 1878, constitue d'une part un document de première main pour qui s'intéresse à l'exercice du pouvoir mayoral dans un village basque sous le Second Empire. Se dessine ensuite, en arrière-plan du témoignage, un portrait de la société locale, dans ses dimensions économiques, politiques, sociales et, dans une moindre mesure, religieuses et rituelles. Autant d'indices qui autorisent, sous réserve d'investigations plus approfondies, une première approximation de l'ordre institutionnel local tel qu'il pouvait se déployer dans cette partie du Pays Basque. Enfin, le manuscrit est rédigé en langue basque. Faute de compétences, l'on ne s'attardera pas sur un commentaire proprement linguistique. Le texte, sous cet angle, attend son exégète. L'on soulignera simplement, d'une part, le recours à une

1 - Ce texte introductif reprend et augmente des éléments exposés lors du colloque Sociabilité et politique en milieu rural, CRHISCO-Université de Rennes II, Rennes, 5-6 juin 2005, à paraître dans : ITÇAINA Xabier, « Pouvoir local, réseaux notabiliaires et sociabilités coutumières. Un maire basque sous le Second Empire », in MISCHI Julian, ANTOINE Annie (dir.), Sociabilité et politique en milieu rural, Rennes, Presses universitaires de Rennes, 2007. Je remercie Julian Mischi pour ses commentaires précieux, et la famille Etchepare à Itxassou pour m’avoir fait découvrir le texte de G. Berrouet. 
langue simple et directe, très proche de la langue parlée, dont les formulations n'excluent pas l'emprunt au vocabulaire français, surtout en matière administrative, tout en déployant un lexique basque très spécialisé (travaux de charpente, de voirie, pastoralisme, etc.). Le choix du basque informe, d'autre part, sur la fonctionnalité de la langue au sein du village, des délibérations orales du conseil municipal, et de la transmission « au son du tambour » (atabal soinuz) de ces délibérations à la population. Tout laisse à penser, enfin, et l'emploi du basque irait dans ce sens, que ce texte construit en forme de justification et destiné aux archives municipales, s'adresse avant tout à des villageois en attente d'éclaircissements sur dix années de politique municipale.

L'on entend ainsi, par la publication de ce témoignage, proposer un matériau empirique susceptible d'alimenter de nouvelles recherches de sociologie historique du politique, notamment à partir du cadre villageois. En terrain basque, le village a servi de support empirique à des études majeures relevant de perspectives aussi distinctes que complémentaires : approche synthétique et historique dans l'analyse du pouvoir politique à Baïgorry (Basse-Navarre) ${ }^{2}$, anthropologie holiste dans l'étude des systèmes de réciprocité à Sainte-Engrâce (Soule) ${ }^{3}$ ou des rituels funéraires et des structures de voisinage à Murélaga (Biscaye) ${ }^{4}$, anthropologie politique de la violence politique à Itziar (Guipuzcoa) ${ }^{5}$, ethnographie économique inspirée de J.-M. de Barandiaran à Ainhoa (Labourd) ${ }^{6}$, etc. Mais au final, ces recherches précieuses mises à part, peu de travaux tirent à ce jour profit de la profondeur chronologique et thématique qu'autorise paradoxalement un cadre spatial et empirique aussi réduit. Les travaux sur le Pays Basque auraient en ce sens beaucoup à gagner d'une sociologie historique maîtrisée du politique, dont les apports ont été décisifs pour comprendre en particulier les processus de politisation des campagnes. L'on rejoindrait ainsi les travaux de l' "école pyrénéenne» de sociologie historique (C. Thibon, E. Lynch, J. F. Soulet, P. Mac Phee, etc.) qui, à partir du cadre villageois, valléen, départemental ou autre, parviennent à réinscrire dans la durée des usages auxquels une certaine anthropologie prête parfois une intemporalité abusive et, inversement, à intégrer la part des mentalités et des sociabilités dans une histoire non réduite à sa dimension événementielle. L'histoire sociale des petites exploitations dans les villages d'Hélette et d'Ascain au $\mathrm{XIX}^{\mathrm{e}}$ siècle telle qu'elle a été dressée par M. Bacqué-Cochard ouvre une voie en ce sens $^{8}$.

Dans le texte manuscrit, l'auteur revient sur dix années de politique municipale entre 1860 et 1870. Tout au long de ces 75 pages et de ces 21 chapitres, Guillaume Berrouet (1816-1890) reprend systématiquement la plupart des actions de la municipalité en matière de voirie, de reboisement, de réparations de l'église, de traités de pâturage transnationaux (facéries), de budget et d'instruction publique. Le village d'Itxassou, situé à 30 kilomètres de la Côte basque, à la frontière de la

2 - BIDART, Pierre, Le Pouvoir politique à Baïgorri, village basque, Bayonne, Ipar, 1977.

3 - OTT, Sandra, Le Cercle des montagnes. Une communauté pastorale basque, Paris, Comité des Travaux Historiques et Scientifiques, 1993.

4 - DOUGLASS, William, Death in Murélaga, University of Washington Press, 1970 (trad. espagnole : Muerte en Murélaga, Irun, Alberdania, 2002).

5 - ZULAIKA, Joseba, Basque violence, metaphor and sacrament, Reno, University of Nevada Press, 1988.

6 - DUVERT, Michel, Trois siècles de vie en montagne basque : Ainhoa, Bayonne, Saint-Sébastien, Elkar, 2004.

7 - DELOYE, Yves, Sociologie historique du politique, Paris, La Découverte, 1997.

8 - BACQUE-COCHARD, Martine, Petites exploitations rurales en Pays basque français (1850-1900), Thèse pour le Doctorat en Histoire, Université Lumière-Lyon 2, 2004. 
Basse et de la Haute-Navarre, compte en 1866280 feux et 1434 habitants. Situé dans le piémont pyrénéen, le village repose alors sur une économie rurale (essentiellement pastorale et artisanale ${ }^{9}$ ) fondée sur un socle coutumier encore en pleine vigueur. Centré sur le travail politique du maire, le manuscrit Berrouet fait émerger deux figures de la médiation politique, l'une dirigée vers l'extérieur et les réseaux politico-administratifs, l'autre s'attachant à réguler les tensions internes à la communauté. On se penchera successivement ici sur la figure du notable entrepreneur, avant d'aborder le rapport du maire aux sociabilités coutumières, autant sur le plan des activités rituelles que sur celui des modes de régulations économiques.

\section{La figure du notable entrepreneur}

Le manuscrit déploie un premier faisceau d'indices autour de la nature du travail politique effectué par le maire d'Itxassou. L'entreprise politique locale s'articule ici autour d'une conception élitiste et notabiliaire du pouvoir local d'une part, d'un indéniable savoir-faire en matière de mobilisation de réseaux et de filières politicoadministratives ensuite.

\section{Élitisme et notabilité}

Élitiste, Guillaume Berrouet l'est lorsqu'il retrace les procédures décisionnelles municipales, qui suivent une filière complexe de consultation des autorités administratives et politiques, du conseil municipal et des propriétaires les plus imposés. Le maire reste éloigné d'une mentalité démocratique, ne dissimule pas son mépris à l'égard des pratiques clientélistes des députés en campagne et souligne l'hypocrisie supposée de certains candidats au poste de maire. La procédure électorale, pour Berrouet, est synonyme de démagogie : ainsi n'hésite-til pas à brocarder son principal adversaire, Martin Teillery, qui, de par sa position d'aubergiste (à Elizaldia), serait poussé à accorder des faveurs à ses soutiens potentiels. La légitimité du premier magistrat de la commune ne vient pas tant, dans cette perspective, d'une légitimation populaire (il a été nommé par le préfet en 1860), mais bien de son action et d'un statut le prédisposant à une perception raisonnée de l'intérêt général. Berrouet, en ce sens, est bien un notable, au sens de $\mathrm{J}$. Lagroye ${ }^{10}$ : désigné maire en raison de sa position sociale - acquise grâce à l'émigration, lui-même étant issu d'une famille modeste ${ }^{11}-$, il dispose de ressources personnelles importantes et exerce une profession lucrative (propriétaire rentier)

9 - L'exploration minière de la montagne se développera à partir des années 1880, notamment sous l'impulsion du géologue Jules Gindre. Une concession sur l'exploitation d'une mine de pyrite de fer fonctionnera au quartier du Laxia entre 1914 et 1954 (voir le texte de Claude DENDALETCHE, «Artzamendi » sur http://www.ateka.eu). G. Berrouet évoque l'extraction du kaolin au quartier Basseboure du village voisin d'Espelette (p. 18).

10 - LAGROYE, Jacques, Sociologie politique, Paris, Presses de Sciences Po et Dalloz, 1997, p. 218.

11 - Son père Etienne Berrouet, fils de bordiers, était métayer avant d'acquérir une maison vers 1816. De son mariage avec Marie Bergés naîtront six enfants. Deux d'entre eux, Guillaume et Dominique, tous deux maires d'Itxassou, bâtissent l'essentiel de leur fortune au Mexique, à Zacatecas. Dominique, propriétaire aisé à son retour d'Amérique (il achète deux moulins, 14 bordes et maisons à Itxassou et quelques unes à Cambo), est élu maire en 1883 et le reste jusqu'à sa mort accidentelle en 1893 (ROUSSEAU, Monique et Francis, Itxassou promenades, Biarritz, Atlantica, 2000). 
qu'il peut conserver durant son mandat. Son approche censitaire du gouvernement communal cependant, bien en phase avec son temps ${ }^{12}$, repose sur une conception patrimoniale du bien public :

«Les maires devraient être pris parmi ceux qui vivent dans l'aisance. Celui qui vit à son aise s'occupera des affaires communes; du moins de temps en temps. Celui qui peine à mener son travail à son terme, celui là ne s'occupera qu'avec difficulté des affaires communes. Ceux-là ne devraient pas prendre la charge de maire. Ils abusent le village et le rendent malheureux, dans le présent et dans l'avenir » (p. 21) $)^{13}$.

Plus loin, le maire réaffirme le principe directeur de son action lors d'une allocution au conseil municipal :

«En tant que membres du conseil municipal, nous devons considérer les intérêts du village comme s'ils étaient les nôtres [...]. Il ne faut pas gouverner le village comme s'il s'agissait d'une maison désordonnée [...]» (p. 56).

Une telle conception, en accord avec la perception paternaliste de la fonction mayorale que J.-F. Soulet repère tout au long des Pyrénées ${ }^{14}$ ne fait pas forcément consensus, en particulier au sein d'un conseil municipal qui repose, quant à lui, sur une légitimité élective. Elle correspond cependant à la politique impériale en matière municipale. La rupture avec la II $^{\mathrm{e}}$ République $^{15}$ avait en effet été marquée par la loi du 7 juillet 1852, qui rendait au pouvoir central la nomination des maires et adjoints (par le Prince-Président dans les communes de plus de 3000 habitants et les chefs-lieux, par le préfet dans les autres). Les maires peuvent, en 1860, être pris hors du conseil municipal élu, ce qui est le cas de Guillaume Berrouet lors de son premier mandat ${ }^{16}$. La pluralité des sources de légitimation politique, que l'on se

12 - Pour une approche de la fonction de maire sous le Second Empire, voir : DAVID, Paul (maire de SaintMatré) , La commune rurale : observations et études, Toulouse, imp. De Savy, 1863 (base Gallica : www. http://visualiseur.bnf.fr).

13 - Les extraits du manuscrit sont traduits du basque par nos soins.

14 - SOULET, Jean-François, Les Pyrénées au XIX' siècle. L'éveil d'une société civile, Bordeaux, Editions Sud Ouest, 2004, p. 129. Voir aussi, pour une sociologie des membres du conseil municipal d'un village basque : LAFITTE-HOUSSAT, François, Municipalité et pouvoir municipal à Ustaritz de 1840 à 1919, Maîtrise d'Histoire, Faculté des Lettres et Sciences Humaines, Université de Pau et des Pays de l'Adour, 1987-1988. 15 - Sous la République, la loi du 3 juillet 1848 décide de l'élection des conseillers municipaux au suffrage universel masculin. Maires et adjoints, pris au sein du conseil municipal, restent nommés par le pouvoir exécutif dans les communes de plus de 6000 habitants et les communes chefs-lieux; dans les autres, ils seront élus par le conseil (AGULHON, Maurice, GIRARD, Louis, ROBERT, Jean-Louis et al., Les maires en France du Consulat à nos jours, Paris, Publications de la Sorbonne, 1986, p. 28-31).

16 - La loi du 5 mai 1855 étend aux communes chefs-lieux de canton la nomination par l'Empereur et fixe la durée du mandat à cinq ans. La loi sur l'organisation municipale du 5 mai 1855 abroge celle du 21 mars 1831 et les décrets des 7 juillet 1848 et 7 juillet 1852, consacre le suffrage universel et accorde un rôle accru au conseil municipal. Le maire, pris ou non parmi les conseillers, fait désormais partie du conseil municipal. Des conditions sont applicables à lui seul : avoir au moins 25 ans et être imposable. La gratuité de la fonction maintenue en écarte toujours les plus modestes. Les élections municipales sont fixées en juillet 1855, et ensuite tous les cinq ans. Les maires sont nommés avant la consultation. Jusqu'en 1865, le gouvernement leur demande de ne pas solliciter les suffrages de leurs administrés, afin de ne pas courir le risque d'être désavoués. L'impopularité d'un homme ainsi désigné est telle qu'à partir de 1865 , le ministre de l'Intérieur invite, au contraire, les maires à se soumettre au contrôle des électeurs et choisit souvent celui qui a été distingué. Il évite ainsi les conflits municipaux qui pourrissent un canton et rejaillissent en conséquences imprévisibles pour un candidat officiel aux législatives. Le tournant libéral du régime est marqué par une circulaire ministérielle, publiée par Le Moniteur du 19 juin 1865, qui finit par admettre que le maire doit être, de préférence, choisi au sein du conseil municipal. Cette prise en considération de l'opinion se perçoit dans la loi du 24 juillet 1867 sur les conseils municipaux, qui modifie celle de 1837. Les domaines réservés au maire s'ouvrent au conseil. Le gouvernement Emile Ollivier (janvier-juillet 1870) met à l'étude un système plus libéral, qu'il ne peut faire aboutir. Voir : GEORGE, Jocelyne, Histoire des maires, 1789-1939, Paris, Plon, 1989 ; AGULHON, Maurice, GIRARD, Louis, ROBERT, Jean-Louis et al., op. cit. 
contente ici de repérer, demanderait à être analysée plus précisément au moment des élections, en tant que moments-clés de l'apprentissage collectif du politique ${ }^{17}$. Reste que cette perception censitaire du gouvernement local est renforcée par l'association constante au processus décisionnel par le maire d'Itxassou des propriétaires les plus imposés, aux côtés des conseillers municipaux. ${ }^{18}$

\section{Réseaux et filières}

En mettant l'accent sur les dimensions entrepreneuriales et volontaristes de son action, le maire compense le déficit de légitimité démocratique par le registre de l'efficacité gestionnaire. Ainsi met-il en marche une topique de la modernisation, passant par une politique des grands travaux et de désenclavement du village et sur la nécessité du soutien à l'instruction (achat de livres, classement des archives municipales, instituteur pour les quartiers de la montagne, etc.). Ce discours se fonde sur des références constantes à la portée " civilisatrice » de la politique impériale de développement économique :

«Les membres du conseil [municipal], tout en voyant que les travaux des routes avaient cessé, ne s'empressaient pas de prendre les mesures nécessaires ; mais moi, en tant que maire, et voulant que les travaux entamés continuent, j'en parlais à la moindre occasion, pour rappeler qu'il ne fallait pas laisser passer le soutien accordé par le Gouvernement pour faire des routes. J'avais beau le répéter, cela revenait au même : ils ne croyaient pas que le Gouvernement puisse faire autant de bien. L'ignorant est incrédule. Le Gouvernement s'était vraiment engagé. Le 15 août 1867 au camp de Chalosse L'Empereur avait dit au Ministre Marquis de la Valette, au vu de l'enquête qui avait été réalisée, qu'il était bien connu que les populations seraient plus civilisées là où l'on ferait des routes. » (p. 18)

Se dégage ainsi une réelle compétence politique en matière d'accumulation de ressources pour la commune. Berrouet est un homme de réseaux disposant de relais dans l'administration et parmi le personnel politique local. Ainsi le voit-on mobiliser ses ressources relationnelles sur les deux grands chantiers de son mandat: la route de Basseboure, entre Itxassou et Espelette, et celle du Pas-de-Roland (athaitzac), point de passage entre Itxassou et la vallée d'Ossés. Sur le premier dossier, le maire mobilise quatre relais : un ingénieur lui sert de médiateur pour atteindre le préfet, et le conseiller général du canton est sollicité pour accéder aux ressources départementales. Le propre frère du maire, Dominique, négociant au Mexique, intervient et fait figure de patron protecteur du village, rendant floue la séparation entre les domaines privé et public. Enfin, les propriétaires du quartier Basseboure acceptent de céder les terrains nécessaires. Relais administratif, politique, familial, villageois-notabiliaire : ces mêmes ressources seront sollicitées autour du deuxième grand projet de Berrouet, la route des gorges du Pas-de-

17 - Voir GUIONNET, Christine, L’Apprentissage de la politique moderne. Les élections municipales sous la monarchie de Juillet, Paris, L'Harmattan, 1997.

18 - Le recours récurrent à la souscription publique (esquia) pour les grands travaux et pour divers achats de la commune (comme des manuels d'agriculture ou de voyage) mériterait également d'être analysé plus avant. Sans doute retrouverait-on probablement les propriétaires les plus imposés parmi les principaux contributeurs, comme le laisse entendre le manuscrit à propos, notamment, de la route du Pas de Roland. 
Roland. Les démarches administratives, politiques et familiales sont toujours parallèles. L'initiative vient de l'ingénieur lui-même, qui fait entreprendre au maire les démarches auprès de l'administration. Celui-ci sollicite parallèlement des « amis » américains, qui lui font parvenir 3000 francs (dont 1000 de son frère), et les villageois les plus imposés. Enfin, la filière accède au sommet de l'Etat. Le maire profite des visites de l'Empereur et de l'Impératrice sur le site du Pas-de-Roland, lors de leurs villégiatures à Biarritz, pour obtenir des ressources. La dernière visite impériale, selon G. Berrouet, remonte au 12 octobre 1867. Il anticipe ainsi sur le virage décisif que constituera l'émergence du tourisme sur la Côte Basque, et évoque à plusieurs reprises l'aubaine que constitue l'afflux d' "étrangers » (estranyerac) à Itxassou. G. Berrouet mentionne à ce sujet l'impact du roman de Jean-Baptiste Dasconaguerre, Les échos du Pas de Roland, publié en 1867. Claude Dendaletche voit fort justement dans cette évocation des aventures romanesques d'une princesse carliste et d'un contrebandier labourdin les débuts de la folklorisation du fait basque ${ }^{19}$. Le maire d'Itxassou saura, en tous les cas, se saisir de ces nouvelles opportunités.

Se dessinent ainsi les contours d'un réseau notabiliaire fondant un ordre institutionnel local stabilisé. Le maire, en tant qu'intermédiaire politique entre la société locale et les différents étages de la sphère politico-administrative, endosse le rôle du médiateur courtier, dont l'activité consiste à rechercher des solutions acceptables entre des groupes éloignés qui peuvent trouver un avantage à coopérer même s'ils ne poursuivent pas les mêmes objectifs et n'ont pas les mêmes intérêts (dimension stratégique de la médiation) ${ }^{20}$. Doit-on pour autant qualifier de clientélaires les pratiques d'échange politique qui sont activées à cette occasion ? Si l'on considère, à la suite de Jean-Louis Briquet, que la notion de clientélisme « sert à désigner des liens personnalisés entre des individus appartenant à des groupes sociaux disposant de ressources matérielles et symboliques de valeur très inégale, reposant sur des échanges durables de biens et de services, généralement conçus comme des obligations morales unissant un 'patron' et des 'clients' qui en dépendent $»^{21}$, alors ces relations de dépendance n'apparaissent pas explicitement ici. La source, cependant, biaise l'analyse. Construit en forme de justification, le texte n'est pas très explicite sur la nature réelle des biens matériels et symboliques échangés entre préfet, conseiller général, ingénieurs, maire, et sur le coût réel de la double médiation, administrative et politique, des courtiers. En revanche, c'est chez ses opposants que Berrouet dénonce des pratiques clientélaires, en décrivant en particulier les relations de patron politique à client entre les éleveurs de la montagne - ses principaux opposants - le député local et le juge de paix cantonal, dont on sait l'importance au XIX ${ }^{\mathrm{e}}$ siècle $^{22}$. Forcément partisan, le manuscrit livre

19 - DENDALETCHE, Claude, « Les échos du Pas de Roland (1867) de J.-B. Dasconaguerre : les débuts de la folklorisation du fait basque ", Bulletin de la Société Ramond, 1997, n¹32, p. 43-46. l'ouvrage de Dasconaguerre a été réédité à plusieurs reprises : DASCONAGUERRE Jean-Baptiste, Les échos du Pas de Roland, Bouhet, La Découverte, 2005. Bien que l'ouvrage soit présenté dans sa première édition comme étant «traduit du basque », il semble que la version basque (Atheka gaitzeko oibartzunak) soit postérieure et redevable à plusieurs contributeurs.

20 - NAY, Olivier et SMITH, Andy, «Les Intermédiaires en politique. Médiation et jeux d'institutions », dans NAY, Olivier et SMITH, Andy (dir.), Le gouvernement du compromis. Courtiers et généralistes dans l'action politique, Paris, Economica, 2002, p. 1-21.

21 - BRIQUET, Jean-Louis, "La Politique clientélaire. Clientélisme et processus politiques », dans BRIQUET, Jean-Louis et SAWICKI, Frédéric (dir.), Le Clientélisme politique dans les sociétés contemporaines, Paris, PUF, 1998, p. 7.

22 - PETIT, Jacques-Guy (dir.), Une Justice de proximité : la justice de paix (1790-1958), Paris, PUF, 2003. 
cependant la trame d'un sous-système politique local articulé autour de relations de clientèles et d'échange politique entre experts, administration et notables. La réelle compétence de médiation du maire Berrouet anticipe sur le type de rationalisation du travail politique qu'Éric Phélippeau ${ }^{23}$ repèrera chez le baron de Mackau sous la $\mathrm{III}^{\mathrm{e}}$ République. Par ses visées modernisatrices et son art du courtage, il préfigure, toutes proportions gardées, et même si le besoin de ressources politiques d'un maire de village du Second Empire n'est pas celui d'un député de la III République, l'émergence d'une figure de l'entrepreneur politique.

\section{Un maire entrepreneur face aux sociabilités coutumières}

Ce travail politique de courtage, essentiellement orienté vers les vecteurs d'un État en expansion, ne doit cependant pas donner l'illusion d'une communauté villageoise monolithique et soudée autour de son représentant. Au travers des attaques incessantes du maire contre ses opposants, ce sont surtout les lignes de partage structurant la politique au village qui apparaissent. Par son action à la fois modernisatrice et reposant sur des bases notabiliaires, le maire entretient un rapport ambivalent aux normes coutumières qui régissent la communauté locale sur le plan des usages et dispositifs tant symboliques que matériels.

\section{Deuils et fêtes : enjeux rituels}

On sait, notamment depuis les travaux canoniques de Maurice Agulhon dans le $\operatorname{Var}^{24}$, tout l'intérêt de la prise en compte des sociabilités festives et rituelles coutumières pour la compréhension des rapports sociaux et de la politisation à l'échelle locale. Les modèles de sociabilité à l'œuvre dans les dispositifs rituels villageois embrassent un spectre de pratiques sociales qui dépasse largement la seule régulation par les sièges déclarés du pouvoir, qu'il soit municipal ou religieux. En matière religieuse, le maire d'Itxassou participe, en accord avec le curé, aux tentatives d'encadrement de la religion locale. Outre, ici aussi, des travaux de réparations à l'église, le maire entreprend de faire éteindre les chandelles de cire funéraire (ezkoak) que les maitresses de maison font brûler à l'église devant la chaise attribuée à chaque foyer. Doit-on voir dans cette mesure, justifiée chez Berrouet par la gêne occasionnée par la fumée, un pas supplémentaire dans la rationalisation d'une religion jugée trop locale ou trop " populaire » ? L'on manquerait d'éléments pour être catégorique. Reste que le geste lui-même prend une teneur symbolique significative dans la mesure où il s'attaque directement au socle juridique et coutumier fondant le lien entre maison, sépulture et église. L'entreprise de rationalisation de la religion locale sera cependant très lente à se généraliser, au vu de la persistance tardive, jusqu'à la fin du XX $X^{e}$ siècle, de pratiques rituelles spécifiques dans ce village ${ }^{25}$.

23 - PHELIPPEAU, Éric, L'Invention de l'homme politique moderne. Mackau, l'Orne et la République, Paris, Belin, 2002.

24 - AGULHON, Maurice, La République au village. Les populations du Var de la Révolution à la II République, Paris, Seuil, 1979.

25 - ITÇAINA, Xabier, «Temporalités rituelles et changement social. La circulation du sens dans le calendrier festif d'un village basque ", dans JIMENO ARANGUREN, Roldán et HOMOBONO MARTÍNEZ, Jose Ignacio (eds.), Fiestas, rituales e identidades, San Sebastián, Eusko Ikaskuntza (coll. Zainak, cuadernos de antropología-etnografía, 26), 2004, p. 315-336. 
On se gardera bien, à partir de ce seul exemple, de caricaturer un maire pourfendeur de la coutume. Le repérage, à l'inverse, d'une attitude bienveillante à l'égard des codes coutumiers rituels dans au moins deux domaines (Fête-Dieu et charivaris) viendrait nuancer cette affirmation. Les notes manuscrites de Berrouet restant hélas muettes à ce sujet, on peut néanmoins recourir à des sources contemporaines du mandat du maire pour une première approximation. À Itxassou comme ailleurs, l'agencement annuel du cycle festif rituel est délégué à la jeunesse masculine célibataire. Celle-ci déploie un savoir-faire en matière d'organisation, de danse et de musique à l'occasion des fêtes cycliques du carnaval, de la Fête-Dieu et des fêtes patronales, et, de façon plus irrégulière, en organisant des charivaris. À l'occasion de la Fête-Dieu, les jeunes hommes composent une escorte d'honneur armée, chargée d'encadrer le Saint-Sacrement durant les processions qui ont lieu deux dimanches de rang, avec danse et musique. Sous la mandature de Guillaume Berrouet, en juin 1863, le sous-préfet, évoquant des conflits survenus l'année précédente autour du commandement de l'escorte, suggère au maire de supprimer la garde d'honneur de la procession, rappelant que « la garde nationale rurale n'existe plus qu'à l'état de pompiers dans les communes pourvues de pompes à incendie, que toute réunion sous ce nom d'individus est illégale et justiciable des tribunaux, à plus forte raison lorsque ces individus sont armés ${ }^{26}$. L'argument est légaliste : sans doute le représentant de l'État voit-il d'un mauvais œil le souvenir des milices paroissiales labourdines d'Ancien Régime. Trois jours plus tard, le souspréfet autorise l'escorte du Saint-Sacrement à la procession de la Fête-Dieu, sur la garantie du maire qu'il n'y aura pas d'incident ${ }^{27}$. La fonction régulatrice et protectrice du maire l'emporte ici sur le volet modernisateur.

Les écarts entre la nouvelle norme étatique et les codes coutumiers s'expriment de façon autrement violente dans les charivaris. À Itxassou, et ce jusqu'au milieu du $\mathrm{XX}^{\mathrm{e}}$ siècle, la sanction charivarique symbolise la $"$ morale des peuples ${ }^{28}$ et constitue un moyen de régulation interne des conflits communautaires. Un sondage aux archives de la commune a permis de repérer treize charivaris entre 1846 et 1890, ou, plus précisément, d'extraire treize cas litigieux entre administration locale, justice et sociétés de jeunesse $e^{29}$. Il ne fait aucun doute que le nombre réel de charivaris pour la même période, largement couverts par la population, ait été nettement supérieur. Ne sont repérés ici que ceux qui font l'objet d'une intervention du juge de paix cantonal, du procureur impérial, du sous-préfet et du maire. Dans la vallée de la Nive, les charivaris prennent, et ce jusqu'aux années 1950, trois formes principales : le vacarme nocturne à répétition au domicile des « sujets » et la jonchée (berdura) entre les domiciles des « coupables », la course à l'âne (asto lasterrak) et, grade ultime de la sanction, la parade charivarique diurne (tobera mustrak), qui peut rassembler plusieurs centaines de participants et fait alterner danse, musique et scénettes jouant publiquement le procès des sujets. George Hérelle $^{30}$ signale la tenue de parades charivariques à Itxassou en 1849, 1865, 1876 et 1883. En 1859, face aux garanties fournies par le prédécesseur de Guillaume

26 -Arch. mun. d'Itxassou, registre «Cultes », Lettre du sous-préfet de Bayonne au maire d'Itxassou, $1^{\text {er juin } 1863 .}$

27 - Arch. mun. d'Itxassou, registre «Cultes », Lettre du sous-préfet de Bayonne au maire d'Itxassou, 4 juin 1863 .

28 - DESPLAT, Christian, Charivaris en Gascogne. La "morale des peuples " du XVI au XIX" siècles, Paris, Berger-Levrault, 1982.

29 - ITCCAINA, Xabier, "Sanction morale, fête et politique : le charivari à Itxassou au XIX ${ }^{e}$ siècle ", Revue d'Histoire de Bayonne, du Pays-Basque et du Bas-Adour, 151, 1996, p. 435-450.

30 - HERELLE, Georges, Études sur le théâtre basque : le théâtre comique, Paris, Champion, 1925, p. 114. 
Berrouet à la mairie, le sous-préfet permet au maire d' "accorder l'autorisation » aux jeunes du village pour qu'ils organisent une fête, "à la condition expresse que la décence, le bon ordre seront sauvegardés et qu'on ne se permettra aucune allusion personnelle $»^{31}$. Or la fête étant en soi justifiée par une « allusion personnelle », seule la protection du maire permet sa tenue. De même peinerait-on à imaginer la tenue de la parade charivarique de 1865 sans l'autorisation du maire. La médiation mayorale, ici, tempère l'irruption de l'État dans la régulation d'une sanction coutumière désormais intolérable pour l'ordre politico-administratif. Le maire protège la jeunesse, dont il sait l'importance en tant qu'institution régulatrice. Cette attitude bienveillante se modifiera sensiblement sous la $\mathrm{III}^{\mathrm{e}}$ République, lorsque les charivaris prendront une connotation politique inédite jusqu'alors, le clivage entre rouges et blancs se greffant sur les divisions claniques. En 1889, le maire Dominique Berrouet, frère de Guillaume, interdit une parade charivarique, celle-ci étant organisée par Martin Teillery, au profil plutôt clérical, et qui s'opposait déjà à Guillaume Berrouet vingt ans auparavant sur les atteintes aux codifications pastorales. Les idéologies ne font ainsi que se greffer sur des clivages anciens directement articulés sur les enjeux socio-économiques et politiques locaux.

\section{De soleil à soleil : enjeux pastoraux}

Certaines mesures prises par le maire Guillaume Berrouet, et avalisées par une majorité très relative du conseil municipal, viennent heurter frontalement les intérêts des éleveurs et bergers de la partie montagnarde du village. La politique de reforestation des quartiers de montagne, en particulier, vient réduire d'autant les espaces de pacages. Le maire entend réglementer le droit de coupe pour mettre un terme aux pratiques de déboisement jugées anarchiques des bergers. Les quartiers de montagne situés au-dessus du quartier Ortzia (Orcia), rappelle Berrouet, étaient encore inhabités quarante années auparavant et recouverts de hêtres (fago ${ }^{32}$. Leur colonisation entraîne une déforestation à laquelle le maire entend remédier, quitte à recourir à la contrainte. Dès 1860, le conseil oblige chaque berger à planter cinq hêtres par an, avec un droit d'usage pour le feu. La mesure entraîne des résistances, et le maire va jusqu'à faire emprisonner un berger pendant six jours, suite à la condamnation par le tribunal de Bayonne pour avoir coupé un arbre à la racine. Les résistances des éleveurs se ressentent également sur la question des prestations «volontaires » pour les travaux d'intérêt général (malobrak). Dès le début de son mandat, en 1861, Berrouet entend compenser l'avantage du libre parcours des troupeaux par une contribution des bergers aux travaux routiers et aux pépinières. En 1862, ces travaux collectifs (équivalents de l'auzolan ou "travail entre voisins " du Baztan voisin) - par quartier - permettent de planter des châtaigniers et 3000 chênes. La réticence des bergers est forte, qu'il s'agisse de la reforestation, des travaux de réparation sur le chemin du Pas-de-Roland suite aux inondations de 1862, ou de l'acquittement du péage sur le pont sur la Nive construit en 1844. Le clivage entre plaine et montagne se fait très sensible sur ces enjeux. D'une certaine façon, les tensions entre quartiers du " haut ", récemment colonisés, et du "bas » du village reproduisent, certes à moindre échelle, les relations complexes et

31 - Arch. mun. d'Itxassou, Lettre du sous-préfet de Bayonne au maire d'Itxassou, 24 juillet 1859.

32 - Les noms des maisons des quartiers les plus reculés témoignent de leur ancien statut de borde dépendant d'une maison de la plaine (Elizaldekoborda, Piarresenborda, Soldatenborda, etc.). 
longtemps houleuses observées dans la vallée voisine de Baïgorry entre les Aldudes, Urepel et Banca d'un côté, les communes du bas de vallée d'autre part. ${ }^{33}$

Mais c'est surtout, en 1869, la taxe pour la jouissance des pacages communaux avec une imposition supérieure pour les montagnards, qui en font une utilisation plus extensive - qui cristallise les oppositions. Le maire justifie cette mesure par le manque à gagner que représente pour la commune l'usage extensif des pâturages communaux par les 8000 brebis, au détriment d'une reforestation bien plus rentable. Le conseil municipal entérine cette décision à cinq voix contre trois ${ }^{34}$. Le clivage entre la plaine et la montagne se double d'une tension entre le maire et les éleveurs. Ceux-ci se mobilisent à plusieurs reprises, contre le bornage des communaux (les bornes sont arrachées) mais surtout contre la taxe sur les pacages. L'opposition des éleveurs est menée par un membre du conseil municipal, Martin Teillery, possédant un cheptel important en montagne. Les bergers ont eux aussi parfaitement intégré les subtilités de l'échange politique local. Ils parviennent à faire annuler la décision du conseil municipal concernant la taxe sur les pacages en faisant intervenir le juge de paix du canton - un valet de l'administration, selon Berrouet - qui entreprend une série de négociations avec le maire et le sous-préfet et rallie le député moyennant un soutien électoral. Les éleveurs renforcent leur légitimité en faisant circuler une pétition dans le village contre la taxe sur les troupeaux. Berrouet n'a pas le monopole de l'art du politique, et le conflit dépasse les limites du village. Les tensions se cristallisent avec le retour au régime républicain, et l'élection - et non plus la nomination par le préfet - par les conseillers municipaux du maire en leur sein. La réforme est présentée par Berrouet comme un appel à toutes les corruptions :

«Lorsque les propriétaires des troupeaux apprirent que des élections se préparaient, une lueur apparut dans leurs yeux, à tel point qu'ils montèrent un complot afin de préparer une opposition pour former un nouveau conseil. Le prétexte était le bornage des limites entre terrains communaux et terrains privés. Ils étaient froissés, car cela concernait les terres qu'ils utilisaient. D'autre part, ils refusaient la taxation des brebis sur les pacages communaux. Les bergers s'unirent par peur de cette taxe. Certains les encourageaient (curayastaçaliac) : un candidat à la députation était passé au village et il leur avait dit : votez pour moi et vous ne paierez pas de taxe sur les pacages. Il aurait eu les voix même sans faire une telle promesse, mais pour ces gens là, peu importe de sacrifier un village, tant qu'ils peuvent satisfaire leurs fantaisies. Et de la sorte, grâce à cette promesse, l'opposition s'organisa plus facilement, et ils se motivèrent entre eux. Face à ces contrariétés, on laissa passer, comme lorsqu'une inondation force un passage » (p. 67-68).

La crise se solde par une victoire provisoire du camp des éleveurs et l'élection au poste de maire de Martin Teillery en 1870. L'attitude réfractaire des bergers face aux atteintes aux usages constitue l'une des « dissidences » que J.-F. Soulet ${ }^{35}$ observe dans les Pyrénées. La dissidence, cependant, loin de s'exprimer de façon ouverte et violente, passe ici par le courtage politique.

33 - BIDART Pierre, Le pouvoir politique..., op. cit.

34 - Arch. mun. d'Itxassou, "Délibération du 7 février 1869 », Registre des délibérations.

35 - SOULET, Jean-François, Les Pyrénées au XIXe siècle, op. cit. 
Le rapport que le maire entretient avec la coutume est plus complexe s'agissant de la question des facéries ou concessions mutuelles de pâturages (traités de compascuités) signées entre la vallée hispano-navarraise du Baztan et Itxassou. Ces négociations transfrontalières, que l'on retrouve sur l'ensemble des Pyrénées ${ }^{36}$, se déroulent directement entre municipalités et/ou institutions valléennes. Berrouet en rappelle le principe de base :

«À chaque fois que les facéries ont été renouvelées avec la vallée du Baztan, ce sont les conditions de 1820 qui ont été appliquées. Les brebis du Baztan sont libres de pâturer ici jusqu'à la montagne du Mondarrain, celles d'Itxassou là-bas sur la même distance, de soleil à soleil, ensuite chacun récupère ses troupeaux. L'on sort des bordes les vaches et les juments, qui peuvent aller à leur guise de jour comme de nuit » (p. 43).

L'ordonnancement juridique coutumier est soumis à une renégociation perpétuelle. À partir de 1847, date à laquelle des facéries sont signées pour dix ans, les Baztandar entendent interdire aux bergers d'Itxassou d'accompagner leurs brebis au-delà de la frontière. Les valléens navarrais réaffirment ainsi leur souveraineté sur les versants et les cols. La tension monte après le renouvellement des facéries du 17 octobre 1865, et les pratiques anciennes de saisie de bétail se multiplient. Face aux difficultés de régulation du conflit par les procédures coutumières, le maire Berrouet fait appel au préfet : celui-ci nomme des délégués, dont l'ingénieur déjà sollicité par Berrouet pour les travaux routiers, et les Espagnols font de même. La chute d'Isabelle II en 1868 en Espagne puis celle de l'Empire en France mettent un terme aux négociations officielles, mais Berrouet signale que les protagonistes du Baztan et d'Itxassou parviennent à un accord oral sur le libre parcours des brebis, sans berger permanent. Pour réguler la crise, les acteurs locaux font ainsi alterner la sollicitation de l'autorité étatique et l'activation des procédures coutumières fondées sur la réciprocité des droits. De même, les conventions empruntent-elles des registres différents : le traité entre institutions locales, le cadre diplomatique pour les relations interétatiques, l'accord oral (bitzez combenituric) entre bergers. La pénétration de l'État et du droit est effective, mais elle compose en permanence avec les modes locaux de régulation communautaire. L'appel à l'État est un recours contrôlé, il n'intervient ici que par défaut. ${ }^{37}$

Le lecteur attentif trouvera dans ce manuscrit bien d'autres thématiques riches en enseignements sur la vie socioéconomique et politique locale : détails du budget communal, emprunts divers, école du quartier du Laxia, état de la population par quartier en 1866, détail des réparations à l'Église, politique de reboisement de la montagne, etc. Au final, le portrait de la société villageoise qui émerge de ce manuscrit est à la fois partiel (parce que partial) et riche en enseignements. L'on se trouve ici confronté à un témoignage situé, cherchant à légitimer une action politique passée. En ce sens, ce texte ne prend sa valeur réelle qu'au terme d'une confrontation des sources qui lui sont contemporaines : délibérations municipales, presse, archives de justice locale, etc. Esquissé de façon embryonnaire ici, ce travail de mise en contexte permettrait à son terme de mieux cerner l'écheveau complexe des relations sociopolitiques dans un village basque sous le Second Empire. Au-delà

36 - On en trouvera une analyse détaillée dans DUVERT, Michel, Trois siècles de vie..., op. cit., p. 191-209. 37 - Sur la pénétration du droit dans les campagnes et la structuration des protestations paysannes, voir les travaux de Jérôme Lafargue sur les Landes (notamment LAFARGUE J., Protestation paysannes dans les Landes. Les gemmeurs en leur temps (1830-1970), Paris, L'Harmattan, 2001). 
de la dimension monographique, la démarche initiée ici consiste à allier plusieurs méthodes pour appréhender la multiplicité des expressions du politique sur une société jusque là trop souvent réduite à des clichés ethnographiques. La transformation des sociabilités coutumières ne prend sens que si elle est située historiquement et replacée dans les échanges politiques et les jeux de pouvoir locaux. Le XIX ${ }^{\mathrm{e}}$ siècle, en ce sens, reste la grande inconnue de l'histoire du Pays Basque français ${ }^{38}$. Tout se passe comme si, focalisés sur des mobilisations identitaires, politiques, linguistiques et religieuses, donc sur des logiques militantes et élitaires, les chercheurs avaient du même coup négligé les formes invisibles et faussement banales du travail politique. Or, les formes de pénétration de l'Etat, en particulier, rencontrèrent davantage de dissidences sourdes, discrètes mais effectives, que d'oppositions idéologiquement structurées, du moins avant la contre-offensive cléricale sous la $\mathrm{III}^{\mathrm{e}}$ République. Descendre au village, en ce sens, permet de rectifier le tir en remettant en cause, par la mise à jour de la réelle complexité d'un jeu politique local étonnamment moderne au milieu du $\mathrm{XIX}^{\mathrm{e}}$ siècle, l'image d'immobilisme parfois associée à la société basque postrévolutionnaire.

38 - Une carence partiellement comblée par GOYHENETCHE, Manex et al., Histoire générale du Pays Basque, $t$. V : le XIX siècle : 1804-1914, Bayonne, Saint-Sébastien, Elkarlanean, 2005. 


\title{
Hamar urthez auçaphezcoa
}

\author{
Guillaume BERROUET
}

Itsasuko auzapezaren (1860-1870) eskuizkribua

\section{[1] Hamar urthez auçapezcoa}

Içan nincen Auçapez icendatua Gobernamenduaz, Itxassuco herrico, Mayatçaren 2an 1860an, M. Orcasberroren ondotic.

Herria hainiz guibelatuya causitcen cen guisa gucietara. Deusic etcen atencionaturic. Herriari tocatzen zaizcon etchiac azken extremitatian ciren. Bidiac bere baitharic demborarekin eguinicaco manera batian. Comunac abandonatuyac, etcen memento agradablia Auçapezcuaren harceco. Herrico erresurxac ttipiac, deusic etçuten munta eguin behar cirenen eguiteco.

Ikhusiric herria cer extremitatetan cen, efort baten beharretan cela, deliberatu nuyen moyanen harcera. Icusico da nola preparatzen nintcen cerbeit eguin behar nuyenian lagunzaren izaiteco. Conseiluyarekin acortian, herrico erresurxac menguatu gabe eguin izan dira seguidan erranen ditudan obrac.

\section{[2] Oihanac}

Ene lehenbicico atencionea izan da arbola landatcia comunetan arthaldiac ibiltcen diuzteneri. Herri huntaco comunen eremuya da 1245 hectara 41 ara eta 63 zantiara. Oihanic ez da calastran 18 ara eta 21 zantiara baizic. Comunetaco lurra arrantan emana dena da cenbeit urtheren 34 hectara, 9 ara eta 70 zantiara larre. Conseiluyac hartuya da deliberacionia hacilaren 11an 1860an, landatceco arçain bacotchac urthe guciez bortz haitz landare comunetan, abar bakhankinen goçatce aldera sucotçat. Eçaguturic yendiaren artian etcela landareric, mindeya herriac hobe çuyela eguitia, guero handic landatceo, eguin izan da Berandotcen, lehenco mindei zaharrian hirur urthez, ekharraraciric ardiez comunac gozatzen diuztenac, non yin baitciren lanera, lehenbicico aldico urrian eta hacirilan 1861an. Oro betan 
ez yiteco, ekharraraci ciren lehenic Errobicuac, [3] Basaburu eta Guerastoco cuartierrac. Bigarren aldian Orciacoac eta Berandotce fronterarainocoac. Hirurgarren aldian Arratecuac, Guibelarte eta Içoquico cuartierecuac. Yendia ez baitcen usatuya deusen eguiten herriarentzat, halere bilcen zen languilia. Ondoco urtian ere eguin cen eraitza frango ezcur eta gaztena eta ere landatu hirur mila haitz landare Espainiatic ekharriac Azpilcueta deitcen den herritic.

Mindeya eguin cen conprendiaraciric behin baino guehiagoetan explicaturic bakotcharen obligacionia, eta noiz. Igande egunetan, Eliza borthan meza erran ondoan. Baitciren bakhar batzu izan etcirenac lanera lehenbicico hasi guinenian mindeyian, huna cer publicaraci nuyen berce explicacionen artian, hacilaren 10an 1861an :

« Azceneco laneco aldian bakhar batzuc hutx eguin dute. Gauza ttipi batetaric escapatceco ez dezazuyela eracusterat eman zuyen borondate escasa. Hitz emaiten diote yaun Auzapeçac, handiagoac harrapatuco dituela [4] lanera yiten ez direnac. Ohart zirete acindaric ez balitz oihanac bere baitharic yin litazkela comunetan. »

Guibelarteco partian ere izan zen pensamenduya mindeyaren eguiteco. Utci zen guerorat hainitz afera izanez herrian eguiteco, eta ere, maiz behartcen guinelacotz populuyaren, diruz edo lan borondatezcuez.

Oihanic ez da guti baicic comunetan. Berandotzen fronteralaino da arbola heldu den lecuya, particularzki fagua. Orai duela berrogei urthe, Orciatic goitian etcen familiaric bici. Demboran hartan bacen abondantcian fago egurra. Oihanac gutituz dohatci, familiac emendatu direlacotz eta ez arthatu faltan. Herrico cargudunec etçuten casuric eguiten arbolen trataciaren gainian. Uzten zuten picatcera bacotcha bere aisiala, suco eta orobat hainitcetan obretaco. Ordria emaiteco arbolen gainian abisatu nintuyen arçainac, ontxa trata cezatela arbola. Bethi libertate osuan usatuyac, etçuten casuric eguiten. Ecin erreusituz erranez [5], hasi nintcen procéberbal eguiten eta desobedient bat atchicaraci preso sei egunez, Bayonaco Tribunalac condenaturic ceren arbola ondotic picatu zuyen. Gueroztic yendia bere baithan sarthu cen, eta arbola ondoco seguidaco yeneralian ontxa uzten zuten. Ordrea eman zen fago gazteric onduaren erditaric ez picatceco, fago çaharreri ez picatceco adar guciac, eta defendatu ciren cerracuyaric eguitetic fagoz. Cerracuya eguitean destruitcen zuten oyhana, adarrac picatuz fago chedarratu berrier. Ordre puscabat eman cen oihanaren tratamenduyaren gainian eta abiatu, ontxa yohan cela beguiz eçagutceco guisan.

Arzainec malobrac eguinarazten zaizcoten fronteraco bide principalian, uda azkenian. Beren goiceco lanac eguin eta yiten ciren seinalatu lekhura lanera. 


\title{
Faceriac acienden alhapidearen gainean
}

Faceriac eguin guintuyen Baztango Alcatiarekin $\mathrm{D}^{\mathrm{n}}$ Juan Bautista Gortary, bortz urtheren, Urriaren 11an 1860an Urdazourin. Bethico usariuetan. Bi kilometre [6] pasaturic libro ciren acindac Espainiacoan. Orobat heyenac Franciacuan distancia berian. Ardiac bi partetacuac iguzkiz iguzki erretira bacotchac bere lekhuetarat.

\begin{abstract}
Athaitzac
Uhalde haundi bat eguin zuyen Mayatçaren 14an 1862an. Hainitz lekhutan etcen eçagun bideric izan zenetz ere. Hobequi conprenitceco cembatetaraino fonditu zuyen athaiz guzia : portale motchetic eta athaitz-burura eguerdico partera etzuyen harri bat utci urac yuan gabe. Orduco Sous-Prefetaren ganic ecin ardietxi guinuyen sokhorriric bidiaren erreparatceco, choilki bide ttipietaco malobrac eta bidetaco diruya soberatua zena 266 libera 32 zentima. Dembora hartan bidiac gobernatuac ciren Agens-Voyerez. Hainiz penaturic, urez bertçaldetic harriac hautxiric, cantitatiac pasatuz chalantian, antolatu zen asqui moldez, languile borondatezcuen laguntzarekin. Pentsatceco da azkenerat zein penagarri zen bidiaren arranyatcia. Continualki uhalde entrealeco [7] guciez eguiten zuyen malurra, cembeit lecutan bidia hartcen zuelacotz. Ahal ciren harriac bilduyac ciren, etcen arranyatcecoric harrapatcen urrun cherkha yuanic baicic. Yina cen dembora ecin entretenitcecua, nahiz hartan emplegatzen ciren bertce legutan emplegatu behar ciren moyanac. Tracazu handia eta despendiua emaiten zuyen bide horrec herriari. Mendy alderdico yendec ecin ekharraraciz lanera trabailuya frango emaiten zuten, nahiz obligacionia zuten ; ecen hortaco barcatcen zangoten abere lana malobretacoa, condicionerekin Athaizetarat yinen ciren bidia arranyatu behar cen aldi guciez.
\end{abstract}

\section{Eliza}

Eliza cen pietate eguin garrico estatu batian. Dorria idekia, continoco citoizez zureria ustelcerat emana. Galeriec etzuten aski soportu, zur nagusiac murruco aldetic tatchatuyac, colomac galdeiten cituzten, hala eçarri ciren beçala, khenduric punçoin mendre lehenago eçarriac cirenac. Galerietaco dornadurac dena [8] cilo handiz betiac ciren. Calostrac ustelduyac oro alchaturic erreparatu behar izan ciren. Iduri zuyen Eliza eguinez gueroz etcela behin ere erreparatuya izana.

Ikhusiric cer estatutan cen, arranyatu gabe ecin utcia cela eta budjetian moyanic ez, posicione horrec icituric utci izan zuyen ene aintcineco Auzapeçac cargua. Mementua cen cerbeitetara hirriscatu beharcena. Egun batez bilaraci nintuyen hiruretan hogoi proprietario seroren etchera compreniarazteco certaco cen orduco bilzarria, huna seguidan eçarria cer erran zangoten oxailaren 23an 1862an : 
«Jaunac, ikusiric Eliza erreparacione beharretan dela eta herriac ez içanez moyenic, Gobernamenduyari galdeguin izan da sokhorria. Badu hilabete bat errecebitu dugula arrapostuya eta dio : herrico Elizaren arranyatceco ditugun erresurxen declaracione bat bidal dezagula, conseiluyaren eta Fabricaco membruen acortian. Guero behar duyela Departamenduco Architetac lanaren gainian debis bat eguin. Formalitate horiec bethe onduan [9] Gobernamenduyac emanen duyela, herriac emaiten duyen lauetaric bat. Herriac moyanaic ez duyen beçala balu ere, Architetac yan leçaque lan suerte hortan Gobernamenduac eman lezaquena seguric. Compreni çaçuye beraz herria obligatuya dela Eliçaco erreparacionen eguitera. Conseiluyac, Fabricaco membruekin acortian deliberatu du, eske bat eguin behar dela, contribucionetara emanic baino yendiac hobeki hartuco duyelacotz. Esperanza dugu yeneroski prestatuco den yendia. Ekharraci ciuztegu ere ikhus deçaçuyen Eliza cer estatutan den, eçagutuco duçuye ez ditekela guehiago atchic erreparacioneric eguin gabe. Içanen duçuya borondatia igaiteco dorreraino aldian sei presuna. Oro idekia da, leihorric gabe, ustelcera çureria emana. Galeriac behar dira solidoki eçarri eta Eliza zola ere peachatu. Hirur mila liberaren empleguya bada ; erraiten dautçuegu ez içaiteco uzcur emaitçaren gainian. Cayer bat eguinen da eta izanen du buruyan Conseiluyaren eta Fabricaco membruen emaitza. Herria currituco du cayer berac, [10] notac hartcen direlaric norc cembat emaiten duyen. »

Hanitzac izan ciren dorrian. Ikhusiric necesitatia bazela, eskia eguin behar cela oroc aithorra eman çuten.

Demboraric galdu gabe eguin cen lista bat, herrico proprietariuena eta taxa indicatu bacotchac cembat eman behar çuyen, hola hobeki erreusitceco. Oroc eman çuten taxatu çangotena. Presuna beharrac ere prestatu ciren, beren heinian, eta bildu izan cen 2460 libera. Dembora hartaco suma aski considerablia, etcelacotz dembora hartan oraino herrian Ameriketaric yinic. Erreparacionen devisa eta plana eguitiaz cargatu cen M. Hiribarren Martin, arpenteur géomètre, eta lanaren executatceco M. Pierre Anchordoqui, conseiluco membrua. Dorreco çureria ekharri cen Berandotcetic eta eçarri lehen baino gorago, forma berian, arduasaz estaliric. Lehen harlauza gorriz cen estalia, hainitz aphal emaiten çuyen begui colpera. Galerier eçarri çaizcoten colomac, ekharriric gayac [11] Athinetic fabricari tocatcen çacon arboletaric. Galerier ciluac tapa eta yar alkhiac arranyatu. Calostrac alchaturic beren lecuyan eçarri non lehenbicio paris puntac Elizan orduyan ezarri beitciren. Eliza ceruya mahain sainduyaz guibeleco aldia erreparatu eta thindatu cen. Barneco paretac oro guisuz churitu. Urthe bat lehenago hasiric thindaraci eta doraraci çuyen yaun Erretorac M. Salaberric, mahain sainduyaz aintcineco aldia. Lehenic thinduya eta doradura carracaturic khendu çacon. Icusi cen oro zur finez eguinac direla. Escultura guciac çurian eguinac eta ontxa conserbatuyac. Alda aintineco colomac peça osoz eguinac dira. Figurac zur berrian atheratuyac. Lan horiec eguin eta iduri çuyen yendiac indiferenciarekin icusten cituyela, halere estimatcen çuten eguin ciren obrac. 
Conseilyuac satisfamenduya emaiteco Eliçan [12] eguin ciren lanen gainean. Deliberacione bat hartu çuyen ontxa eguintçat hartcen çutela Auçapeçak eguin araci obra eta seguidan eman den despendioa, eta oroc sinatu deliberacioneco liburuyan Oxailaren 8an 1863an.

\begin{tabular}{|l|l|}
\hline Eliça ceruyaren thindatcia eta erreparacioniac & 650 \\
\hline Dorreco arduasa bere lecuyan plaçaturic & 440 \\
\hline Çureria eta taulak Anchordoqui fornituyac & 795 \\
\hline Oficialen yornalac & 300 \\
\hline Harguinenac & 80 \\
\hline Bertce gaineraco fornimenac & 390,75 \\
\hline Totala & 2653,75 \\
\hline
\end{tabular}

\section{Herrico Recueillac}

Herrico errechistruac eta recueillac, erran nahi baita Prefeturatic yiten diren Gobernamenduco ordriac eta leguiac ciren molde tzarrian, paketa listaz escatuyac, ordreric gabe, denac [13] herrautx, begui colpe itsusian. Bertze hainitz liburu ciren achalac hautsiac, nola calastraco planac. Denac achal berriac emanic eçarri ciren Bayonan, liburuca numeratuyac, urthe gucietacuac, erresqui behar orduyan harrapatceco guisan.

Denbora berian haurren escolaco alkhi eta mahainac eguin ciren eta çaharrac arranyatu. Haur pobrentzat liburuyac erosten ciren. Hortaco bujetian eçartcen cen suma bat, behar arabera ekhar eta emaiteco haurreri.

\section{Elizaco cutchac eta arguiac}

Eliçan baciren cutchac emaztekiec beren tapicen ezcuen ontci cerbitçatcen cirenac. Etche guehienec baçuten beria eta ciren handitasunian liferentac, eguiteco manera ere ba, baciren goiti idequitcen cirenac. Embrazu handia emaiten çuten, nahiz murruyari contra ciren eçarriac, erortcen noiz nahi, ceren baciren asco lekhutan hirur eta lauetaraino elgarren gainian. Egun [14] batez Erretorac, Monsieur Darraidouc, desgustatuya, denac ereman cituyen sala handira. Yabiac etciren content. Oroc ekharri ciuzten beheiti, leheneco lekhuyetarat. Cutcha berriac eguinaraci behar cirela oro peza batetan proposatu nion Conseiluyary, eta seguidan yendia abisatu guinuyen. Huna cer erran çacoten Urriaren 25an 1862an Eliça borthan :

"Ikhusiric yaun Auçapeçac herrico cargudunequin batian, Eliçaco cutchen nombria hain handituya dela, hainbertcetaraino nun ez beita lekhuric aski traburic eguin gabe plaçatceco, deliberatu dugu cutcha berriac eguin behar direla, orai bertciac diren lekhuyan peza batetan bertce hanitz herritan diren bezala. Emanen 
dira bacotchari bere yar lekhuyari dohazcon guisan, numeratuyac, gakhoac eskura. Ez da bakhotcha gostaco hirur libera baicic. Egun çortci meçac ondoan hartuco dira etchen icenac. Nahi duten guciec eguinen [15] dute galdia."

Orori ontxa idutiru çangoten eta berheala hartu ciren moyanac cutcha berrien eguinaraztecuac. Leheneco çaharrac yuan cituzten beren etchetarat eta 174 cutcha berriren distribucionia eguin cen igande batez Marchoaren 28an 1863an.

Denbora hartan emaztekiec usatcen zuten Eliçan beren antcinean ezco piztuyac. Trabu handia eguiten çuten arguiec; hainitz aldiz erretzen ere arropac elgarri. Foncionetaco demboran yendia incomodatcen çuten, arguiec emaiten çuten khea cela medio. Yaun Erretorarekin concertaturic khen araci behar cirela, compreniaraci cion populuyari usaya galdu behar cela arguien aintcinean atchikicecua. Ongui handia eguin cen yendiaren descansuya gatic Eliçan.

\section{Hilerriac}

Seguidan emaiteco Eliçari tocatcen çaizconac. Hilerriac ciren idequia yenden orhoit gucian. [16] Harresiac hainitz lekhutan erditara yinac, eta sarce guciac portaleric gabe. Lekhuyaren gaineco animale suerte gucietaric hilerrietan ikhusten ciren, particularzqui cherriec eguiten çuten calte handia. Malurac guerthatcen ciren eta huna certaco. Lehenago hainitz hilerrietan portalen barneco aldetic çolan emaiten ciuzten burdin grilac, guisa hortan ciren herri huntan ere. Portaleric etcen lekhuyan, guisa hartaraco sarcia, clar da malurra guertha citakela memento guciez.

Hilerrietaco harrasiac antolatu ciren eta Fabricaco membruekin acortian eguin araci guintuyen burdinazco portaliac, despendiuac herriarekin erdisca. Dembora berian seroren etchia erreparatu cen. Murru mendriac urratcerat emanac ciren. Soleitceco campotic ernayer eman ciren burdinazco y grekac. Gaineco sala tronatu cen eta murruyac emocatu. Eliza erreteilaraci guinuen eta dembora berian [17] herrico etchia erreparatu. Ihaureco çubiari madriga berriac eçarri ciren, ekharraraciric gueyac Berandotcetic.

\section{Espeletarat bidea}

Aintcineco erran ditudan obra horiec eguitian orhoitzen nintcen herriac etçuela bide berriric eguinic nehorat. Bide haundia ${ }^{\circ} 19$ cen eguina, lurrac herriac emanic, 1840etic eta 1845 artian. Herri huntan orai dino nehor ez da ocupatu bide eguiten. Etcen nehon çaldi bati calupa puscabat eman citeken bideric. Bethi danic malobra bidetacoac eguiten ciren molderic gabe, ahalic lan gutiena eguinez, orobat behar 
etcen lekhuyetan, hainitz aldiz manaçaileric gabe, hain molde gaizqui eguiten ciren non ikhusi baitira, tumberuac hostoz estaliac, combeni etcelacotz icustia barnian zuten legarra. Bide çaharretan arroila cembeiten eguiten hassi (sic) berriac ciren basuren istant baten khenceco.

Cantonamenduya, Espeleta, etcen bideric Urçumun [18] gaindi baicic, çaldizcuen eta oinezcuentzat. Bidiaren plana bacen eguina Basaburun gaindi 1848an Monsieur Hiribarren eta Prefetac aprobatuya. Hirur herric behar zuten bidia eguin, hasiric bide handitic $\mathrm{n}^{\circ} 19$ eta Espeletaco carricala. Itxasuco herriac erdia, Espeletac ehuneco berrogoi eta Luhusoc ehuneco hamar. Dembora hartan Espeleta Baseburun baciren fabricac kaolinaren trabailatceco. Nagusiec pusatcen zuten bidia eguitia nahis, beinan herri hunec seguric etçuyen moyenic. Arrazoin horren gatic utci çuten hasi gabe bidia ene aintcineco hirur Auçapecec. Etcen facil eguitia, 4048 metra luce baçuyen herri hunec eguiteco. Bidiac hartcen çuyen lurraren indenitatec munta çuten 5500 libera, eta santima bat ez pagatceco erraiteco maneran. Gu ere situacione berian causitcen guinen. Ikusiric dificultate horiec 1861an examinatu guinuyen gastuetaric eta indenitatetaric escapatceco bacenez moyanic. Macharretic [19] Celhaico ondoa beheity hartuz eguin othe citeken. Espeletarat yausteco patar sobera causitcen cen. Etcen berce moyenic Basaburutic eguitia baicic. Abiatu guinen lehenic bidiaren ikhusketan, maiz plana eguilia M. Hiribarrenekin. Lekhu gehienetan bacen bidia (en état de lacune) naturalki eguin estatuyan. Errecartetic Haranera lehenago içana cen bidia oraico lekhutic, galduya cen, etcen orgaric pasatcen ahal. Haranetic Espeletaco alderat etcen bideric. Mintzatcen nituyen lurren yabiac, yaquiteco desiratcen çuten bidia eguitia. Batian erraiten çautaten bayetz, ondoco piayan orobat ez. Bi urthe pasaturic yan ciren indenitateric pagatu gabe lurren ardiesteco. Cer desgustuyac izaiten nintuyen hainitcetaric ! Batzuyetan elhe gaitxes garriac. Beinan ahantzten ciren cerbeit eguin nahi nuelacotz. Azkenian eman ciusten lurrac compreniaraciric etcela bideric bercenaz eguinen. Batzu hasi ciren emaiten eta guero heyen exempluya seguitu çuten bertzec. [20]

Dembora hartan Ponts et Chaussetaco Inguignadorearen carguco ciren bidiac. Arrondisamendu huntaco cen Monsieur Boura. Egun batez Saran guerthatu guinen etche berian. Ezagutçac eguitiarequin erran nion bidez ocupatu nahia nincela. Desiratcen nukela ene etchera yin baladi seguidan biden examinatceco. Etçuyen dembora handiric pasatu yin gabe. Içan guinen bide intres communaren icusten $\mathrm{n}^{\circ}$ 49. Examinatu zuyen plana aspaldian eguina, lekhuaren gainian comparatuz. Errecaco etchia barna norteco aldetic cen bidiaren plana hartuya. Etcelacotz guisa, orai bidia eguina den lekhutic traçaraci çuyen. Itxasuco partian, Errecartetic fite hasiric, Haraneco etchiaren guibeletic cen traçatuya penciac barna. Oraico lekhutic ardietxi cen nahiz bacen patar sobera. Indenitatiac [21] ez pagatceco eguin cen modificacionia. Bidiaren eguitia neholere necesario cela orok ikhusten zuten, ez choilqui herri hunentzat baicic ere urrunagoco piayian çabiltzanençat. Cambon gaindi çabilan yendia carrosez edo orguez behar cenian baliatu, eta dembora hartan nolacoa cen Imbidiaco erreca ? Ecin pasatuya.

Moyanek falta eguiten çaucuten bidiaren eguiteco Basaburun gaindi. Erran çaharra ensayatu gabe ez dela deusic izaiten, ez eta ere erreusitcen irriscatu gabe. Hemen erran behar dut gauça bat : herrietan Auzapeçac behar litezque eguin aisian 
bici direnetaric. Aisian den guiçona ocupatuco da herrico aferez; bederen noician behin. Bere lanac ecin eguinez penatcen den hura, herrico aferez arrarozki ocupatuco da. Holacoec ez lukete hartu behar Auçapezcua. Herria abisatcen eta malurusten dute presentian, bai ethorkiçunian ere . [22] Erran nahi baita deusic ez eguin eta cerbeit eguin leçaquetenac empechatcen diuztela deusic eguitetic. Comparacione bat behar dutenean herri hunen gainian ez hala guerthatuco delacuan aguian. Lehenago conseiluya hainitz ocupatcen cen mindei baten eguiteco Berandotcen, icusiric hainbertce lur alfer baduela herriac hetan landatceco. Ya badira haitz landariac asqui handiac eta seguidan içanen zazpi edo çortci mila, ecin estimatuyac lecu hartan. Malur handia litaque herriaren (sic), mindeya eguin cen bezala bil araciric yendiac landatzen ez baluzte.

Ikhusi nuenian esperantza bazela bidiaren eguiteco, batetic lurrac urriric izaiteco, esperantza eta Gobernamendutic socorria izanen nuela izquiribatu nuyen ene anaya Domingo Zacatecasera (Amérique) erraiten nioyelaric nola ocupatcen nintcen Basaburun gaindi Espeletaraco bidiaz, sokhorri beharretua nincela. Ene idera complitu çuyen. Bidali zauzkitan mila eta bortz ehun libera suscripcione baten medioz bilduric herritarren artian. Ya bacen arrazoina Gobernamenduayari sokhorriaren galdeiteco. M. Boura Inguignadoria yaquinsun eguin nuen. Erran çauntan mila libera izan arazico zauzkitala Prefetaren ganic.

Isquiribatu nioyen M. Goyetchi, cantoinamenduco conseilu yeneraleco membrua, baliatciaz ere izanarazteco erran mila liberac. Hainitz ongui prestatu cen eta ardietxi esperantza cena. Berac ere eman cituyen hirur ehun libera.

Bidiaren idequitzeco erresurxac bildu ahal ciren becen fite, nibelamenduya hasi Urriaren 2an 1864an. M. Hiribarrenez, eta escu lana Martchoaren 5an 1845an, regiaz, taxa emanic, herrico languiler. Bilcen ahal ciren erresursac eguin ahala bilduric ere etcen batere sobera. Hortaco [24] bide handico malobrac galdeguin ciren Basaburuco bideco, conseiluyaren deliberacione baten medioz eta ardietxi guinituyen. M. Boura cen causatcen çuyena emaitia edo errefusatcia, certaco Préfeturatic yiten zaizcun biden gaineco conseiluyen deliberacioniac.

Ontxa pentsatuz eta moyanen izaitera hainitz enseyaturic huna cer erresurxen gainian khondatzen guinuyen Espeletaraco bidiaren eguiteco Basaburun gaindi :

\begin{tabular}{|l|l|}
\hline Errecibituya diruya Ameriquetaric & 1500 \\
\hline M. Goyetchen ganic & 751,30 \\
\hline Prefetac M. Dauribeau, Gobernamenduco sokhorria & 1000 \\
\hline Bide handico ${ }^{\circ}$ 19 malobrac urthe batetacoac & 751,30 \\
\hline Bertze fuilarat pasatceco totala & 4156,30 \\
\hline [25] Bertze fuilatic ekharria & 4156,30 \\
\hline Bide ttipietaco malobrac & 375,65 \\
\hline Bide ttipietaco malobrac, diruz pagatuac & 600 \\
\hline Departamenduco sokhorria 1866an & 500 \\
\hline Ume çurtcen diruya soberatuya & 700 \\
\hline Totala & 6331,95 \\
\hline
\end{tabular}


Abere lana malobretacua cerbitzatu cen lurren kharreyatceco batetic bercera Taxatcen çuten cer cantitate egunian behar çuten kharrayatu. Baçuyen urthe bat emana cela malobrac thaxatuz eguiteco usariua. Gorago eman suma horrekin etcen bidia edequia baicic.

Bidia idequi onduan harristatu cen, ez yendiac desiratcen çuyen becin fite, certaco insuportable baitcen han ibilcia dembora umia cenian.

Ondoco urthetan ere ardiesten guinituyen bide handico malobren parte bat bederen Basaburuco emplegatceco.

Pilotaco plaça arras bardin gaitz cena, [26] arranyatu cen hainitz lur ekharriric nivelatceco, lapitz chehia eçarri gainian, ekharraraciric athaiz burutic. Dembora berian landatu ciren Arrosaco etchiaren aintcineco platanac.

Bide berri gucian pagatu cen indenitatec etçuten munta 500 libera baicic, cerracuyequin 900 libera. Bidiaren eguiteco lurrac eman ciuztenekin, herriac eguin cituyen proceberbalac urriric lurrac emaiten ciuztelaco declaracionea, paper imprimatuyan, ceinac merian baitira alchatuyac.

Espeletari tocatu çacon bidia onduan eguin cen, indenitatiac pagatuz proprietario gucier.

Marchoan 1865an landatu ciren 165 peupliers landare, bide handian n¹9an, bidiaren bi bazterretaric, Anchordoquitic hasi eta seguidan.

\section{Budjeta}

Huna seguidan eçarria Itxassouco herriaren situacionia, erresurtxac, bujeta eguina Mayatçaren 11an 1865an.

Urtheco errebeniua

8925,65

Suportatcen diren gastuyac

8920,23

Herriac baditu bere disposicioneco ekhartcen diren sumac. 


\begin{tabular}{|l|l|l|}
\hline 1. Diruz pagatu malobrac bide handicoac & 222,90 & \multirow{2}{*}{351,29} \\
\cline { 1 - 2 } 2. Zantima adicionala id. & 128,39 & \\
\cline { 1 - 2 } 3. Bide bicinaletacoac id. & 299,52 & \multirow{2}{*}{606,32} \\
\cline { 1 - 2 } 4. Id. malubrac diruz pagatuyac 1864an & 251,90 & \\
\cline { 1 - 2 } 5. Zantima adicionalac bide ttipietacoac & 54,90 & \\
\cline { 1 - 2 } 6. Ume zurtzen diruya cachan soberatuya & 705,39 & 705,39 \\
\hline 7. Urthe huntaco ume zurtcena & 135,13 & 135,13 \\
\hline Totriaren disponible aintcineco urthian gueldituya & 607,88 & 607,88 \\
\hline & 2406,01 & 2406,01 \\
\hline
\end{tabular}

\section{Athaitzetaco bidea}

Basaburun gaindi Espeletaco bidia eman zenian eguiteco. Egun batez languiliac ikhusiric heldu guinelaric erraiten M. Bourac ez nukenez [28] ocupatu nahi Pas de Roland-go bidiaren eguitiaz (Administracioneari lehenago aiphatua guinuyen). Bayetz erran nion, nahiz lan penatu bati hasi behar nintcen.

Erran nion bidia eguiten ahal balitz herriaren abantail handia litekela. Herrico yendiaren bortzetaric biac Athaicez goitian bici direla. (?)reina handia dutela, gauça guciac buruz edo çaldien bizcarrian pasatcerat obligatuyac. Uhalde entrealeco guciec bidia hainitz lekhutan funditcen, aldi guciez herria obligatuya arranyatcera, mendico habitanten medioz, obligacionea dutelacotz ceren barcatcen çaizcoten malobretaco abere lanac. Diferentcia horrek ekharcen duyela herriari urthe guciez hirur ehun liberaren malura. Erran çautan Inguignadoriac herriac eman nahi badu cerbeit, izanaracico çuyela sokhorria fite idequitceco guisan. Informatuco nintzela erran nioyen. Adreçatu nintcen ene Americano laguneri eta fite muntatu cen suma bat hirur mila liberetan pasatcen cela. Ene anaya Domingo [29] berac eman cituyen mila libera. Herrico conseiluya yaquinsun eguin nuyen eta consideraturic moyanen harceco manerac bacirela, comunicatu nioyen M. Bourari yaquin ceçan herria prestatcen cela yenerosquy.

M. Dauribeau Prefetac bil araci çuyen herrico conseiluya, deliberatceco bidia eguitia nahi cenez eta cer erresurx duyen herriac emaiteco. Deliberacionia hartu cen, bortz mila libera suscripcione borondatezcuaren medioz eta escu lana yendearen borondatera. Oro zazpi mila libera. Seguidan eman çuyen ordria Prefetac, bidiaren plana behar cela eguin Bidarraico limitera artino, Harnabaleco gaineco aldera. Plana herriac eguinaraci behar çuyen.

Huna cer publicatu nuyen Eliza borthan, urriaren 16an 1864an :

«Prefetac desiratcen du Athaizetaco bidiaren plana hartcia Bidarraico lurrera heldu artino. Planaren eguiteco gastuyez herria behar da cargatu. [30] Berheun (sic) libera behar da atchiqui plana eguiten deneco. Herrico Auzapheçac mintçatu 
ditu cembeit proprietario diru horren bilciaren gainian. Prestatcen dira gogotic bacotcha bere partiaren emaitera. Athaizez goitian eguinen da diru horren bilceco esquia.

Oroc ikhusten dugu erloiric gabe guirela herrian. Triste eta desohore da gabe izaitia. Erloya behar da arranyatu. Bi urthez nahi du garantitu moldazailiac. Ez badu erreusitcen ez du pagamenduric galdatzen. Itxassou beherian eguinen da erloyaren arranyatceco esquia. Lehen eman duzuyen hiruretaric batez içanen cirete thaxatuyac. Auzapheçac esperantza du lehengo borondate berarequin emanen duçuyela tocatcen çautçuena, ikhusiric ocupatcen dela ecin utzizco herrico aferez. Ez uste izan sobera ocupatcen guirela, batzu bertciac becen importantac dira eta conprenitcen duçuye.

Espeletaraco bidiaz questione, bidia pasatu behar den lecuco proprietariuac prestatcen dira [31] yenerosqui, emaiten diuzte urriric lurrac. Guero badira indar eguiliac, non herria batere behartu gabe bidia eguinen beita. Athaizetaco bidia gure indarrez goragocua da, bainan ez da faltatu behar, ahal arabera laguntça casuya yiten denian. »

Esquia eguin cen eta bildu orotara 416 libera. Bidiaren plana eguin zuyen M. Hiribarrenec. Prefetari bidali eta aprobaturic turnatu çuyen herrirat, ikhus ceçan conseiluyac eta populuyac.

Bilaraci nuyen herrico proprietario cembeit, conpreniarazteco ez dela Gobernamenduya ocupatcen bide horren eguitiaz, yaquin zezaten ahalaren arabera eman gabe etcela bideric hasico, etciten flata Gobernamenduyac eguinen çuelacuan.

Huna seguidan cer erran çangoten Hacilaren 12an 1865an :

« Afera serios eta importanciazco batez ekhar araci ciuztegu hunat, Athaizetaco bidiaz questione. [32] Adituac cirete bide horren equiteco desiretara trabailatcen guirela, lagunduyac izaiteco esperantzan eta ardietxo dugu fagore hori. Moyanac badira eta behar guira baliatu ocasioniaz. Prefetac emaiten dauzcu hasteco bortz mila libera. Orai beretic hirur mila gure cerbitçuco dira. Sokhorri hori eman du Prefetac, ceren conseiluyac declaratu duyen herriac emanen tuyela orai hasteco orotara zazpi mila libera, suscriptione borondatezco baten medioz, eta esku lana harrequin. Herrian dugun plan bidiarena Sous-Préfetac bidalia ; ikhus deçagun eta delibera definituqui eguitera gaudenetz. Ez da Prefeta ocupatcen bide hortaz, herri bidia delacotz. Sokhorria izaiten badugu ardietxico da adisquiden medioz.

Bada emaitza bat Americanuena hirur mila liberetan pasatcen da. Ikhusiric abantail horiec, norc errefusatuco du çuen artian partiaren emaitera ? Ikhusten 
duzue bide hortaz profitatzen ez direnec emaiten dutela suma bat asqui handia. Ikhusten dute [33] herria bi pusquetan dela. Ikhusten dute uhalde guciez ecin peachatuz cembat tracazu eta despendio duyen, bertce bidetan emplegatu behar diren erresurxac Athaizetan emplegatcen direla. Hitz batez erraiteco, ikhusten dute 1855an 1856an eta lehenago aiphatua dena 1862an becelaco uhaldiac eguiten baditu, nequez eçarrico dela oraico estatuyan. Badute consideracionia behar guirela izan batçu bertcen sokhorrizaile, ahal ditaquenian. Erranen çautzue bakotchac cembat eman behar duyen, orai erdia eta urthiaren buruyan gaineracua.

Perceturraren escuetan behar da depositatu emaitza hori. Bidiaren eguitia gostaco da. Halere bentzutuco dugu eta eguinen, gure aincinecuec moyanetara ecin helduz, guticia handirequin egui gabe utci izan duten obra."

Demboraric galdu gabe seguidan eguin cen esquia eta eman çuten bai behereco habitantec, nola mendicuec, bacotchac bere partia. Lanac ciren, orai [34] errechago dira eguinac ikhustea. Moyanic etcen eta obrac eguitecotz behar ciren fondatu. Bonurra enetzat, erreusitu baita eta ororen profituya presenteco eta ere ondocoentzat.

Inguignadoriac, M. Bourac etzuyen nahi demboraric galçadin. Bidiaren traçatzeco piquetac eçar araci cituyen bera present celaric Athaiz gucian Hacirilaren 26an 1865an. M. Longuet eta M. Hiribarrenenen medioz.

Berria hedatu cenian bidia eguin behar cela, hainitzer iduritcen çangoten ez guinuela erreusituco, lan terriblia celacotz ; iduritcen çangoten gure indarrez goragocoa. Ibili ciren entrepreneurrac, bide eguiliac icusquetan ; soinac uzcur eta turnatcen ciren icituric ecin comprenituz lana. Harroca hutxic baicin etçuten icusten, etcitezquen mentura galdeitera cembat behar çuten metra curanteco.

Guretçat ere dificultate berac ciren, bainan hasi behar eta deliberatu guinuyen regiaz eguitera, erran nahi baita yornalean. Dembora hartan [35] ez beitcen herrian minurric ekhararaci ciren herriz campotic. Nesesario ciren tresnac eguin araci ciren ; tarateluyac, mailuyac, palangac eta haintzur picuac. Hain cen harri borthiça, non alceiru urthuya emanez tarateliyetan, halere harrien hausteco dificultate handiac baitciren.

Hasi cen bidia M. Hiribarren surbeillant, Athaiz burutik, orai murruya hasia den lekhutic, Oxailaren 6an 1866an, eta lehembicico mina tiratu 14an, hilabete berian. Bide çaharra oraino murruyaren oinetaric cen, pasaya libro utci behar lana frango emaiten çuyen bidiaren libratcen harrietaric.

Nahiz esperantza guinuyen lagunduyac izanen guinela Administracioniaren partetik, icusiric lan handiari lotcen guinela, adreçatu guinen gure conseilu 
yeneraleco membruari M. Léonce Goyetchi. Bidial guinuyen memorio bat eguina, Meriaco secretariuac, M. Jean-Pierre Berterechec, çoinetan explicatzen [36] beitzanzcon Athaicetaco bidiaren importantcia, bai herrian eta herriz campoco, Prefetari presentatceco sesion ordenarioan. Ondoren ona ekharri çuyen, lagunduac içan guinen eta M. Goyetchec berac eman çaucun 250 libera.

Huna cer erresursa izan cen Athaizetan bide eguiten empleatzeco, çoinarequin idequi baitcen lau ehun metraren inguruya.

\begin{tabular}{|l|l|}
\hline 1866an Aphirilaren 14an herrico habitantetan bilduya & 3414 \\
\hline 1866an Aphirilaren 15an M L. Goyetche & 250 \\
\hline 1866an Aphirilaren 30an Emperadoriaren ganic & 3000 \\
\hline 1866an Buruilaren 19an Prefetac & 500 \\
\hline Abenduaren 6an herrian bilduya & 1687 \\
\hline 1867 Urriaren 16an Prefetac departemendutic & 500 \\
\hline 1867 Emperadorearen sokhorria & 5000 \\
\hline 1868 Abendoaren 28an Gobernamendutic & 425 \\
\hline 1870 Martchoaren 30an Departamendutic & 500 \\
\hline 1871 Aphirilaren 15an Departamendutic & 200 \\
\hline Totala & 15476 \\
\hline [37] Bertze fuillatic pasatu suma & 15476 \\
\hline 1871 Malobra borondatezcua & 1000 \\
\hline Totala & 16476 \\
\hline
\end{tabular}

Espeletaco conseilu errebisioneco egunian Mayatçaren 9an 1866an, Prefetac, M. Darribeau izan zuyen borondatia aceptatceco ene gomita gosaitera. Yin cen bere seguidago yendequin : M. Boura, Inguignatoria, Cunisse, Sous-Prefeta, Dasconaguerre, conseilu yeneraleco membroa, Dihursebehere, Saracua. Athaizetan orducotz minurrec baçuten bide lekhu pusca bat tiroca harrocac alcharaciac.

Ikhusi çuyen Prefetac chantiera eta bide eguin behar cena. Sordiesa çancon beinan etçuyen erran solas lotxatcecoric. Bide handitic, grande communication $\mathrm{n}^{\circ}$ 19etic eta Athaizetara oinez çabilan yendia, etcen posible berce maneraz. Yende estranyera gueroago guehiago çabilan. Emperadoria izan celacotz behin baino guehiagoetan. Berçalde M. Dasconaguerren obrac Les échos du Pas-de-Roland, fama handia emanaraci cioyen. Prefetac ikhusiric bidiaren importantcia habitantenzat eta nola estranyerec [38] hartu zutelacotz afectione bat promenatcerat yiteco, proposatu çauntan carrosa bide bat eguin araci behar nuyela bide handitic eta Athaiz burura. Bortz ehun libera emanen zaizquitala. Sokhorri tipia cen bainan aceptatu nion, erran bezala neholere nesesario celacotz eguitia.

Hemen ez nauque gauza bat erran gabe. Ascotan suertia ondotic ibilcen çaucu ; ez guira baliatcen hainitcetan neglientzeaz edo erreflectione escasez. Ur handico burdinazco çubico pasaya libratu zen 1844an. Zubia eguiten hasi gabe entreprenurrac abisatu zuyen herria, proposatcen çuelaric cer enfort eguin gogo zuyen pasaya libro izaiteco. Fornitu behar cen harria, sablia eta çureria. Orotaric pusca bat forni citaiqueyen. Orduco Auzaphezac, bere funtxian, bilaraci cituyen 
proprietario hainitz cuartier gucietaric. Menditarren aldia yin cenian beren opinioniaren emaiteco, declaratu ciren erraiten zutelaric, heyec etçutela zubia batere profitatuco. Auzapeçac erran behar bidian menditarrec profitatzen [39] çutela beheriarrec profitatcen ez dutena frango, batac bertcia compensatcen çutela. Huna cer erran cioten eta huntan despeitu :

"Ni ere gutitan pasatuco niz eta pasatzen nicenian pagatuco dut aldico sosa."

Zubico tarifa Sous-Prefeturan egon cen asqui dembora, oroc ikhus ceçaten erreclamuric balinbazen eguiteco. Herri huntacoric nehor etcen aguertu. Auzaphez bera ez da libro zubian pasatcera pagatu gabe, herrico aferez yohan bada ere. Hola guidatuyac izaiten dira hainitzetan herriac, ez da estonatu behar izanagatic penatuyac ontxa guidatu faltan.

Erraiteco zein lehku destartac ciren hauc. Bide handitic Athaicetarat estranyerac guidequin yoaiten ciren. Unhatuyac ibilcen ciren bide tzarra celacotz, dena harri eta cilo. Bide handitic eta Uhalde Etcheverrira etcen clasatuya. Cerbitçu guti çuyen bidia cen orduco maneretan. Halirico intha etcen bi metra çabal baicic ; bethi causitcen ciren [40] artho çanguak ustelcerat botatuyac, handic aintcina behinere hainçur colpe bat emaitecua. Uharretuya pasa eta etcitequen izan lehku tzarragoric, dena ur eta liga, pausu gaichtuac ciren eguiteco.

Bide berria Athaizetara hasi cen Mayatcian berian, malobren medioz eta izan cen borondatezcua frango, galdia eguin eta Errobico cuartieretic eta Olasurretic. Erran behar da yendia prestatcen cen. Icusten çuten progreco marcha bat herrian behinere izan etcena, horren medioz curayestatuyac obeditcen zuten. Bidiac hartu cituyen lurrac proprietariuec urriric eman ciusten. Uda berian bidia eguin cen Athaitz burura. Nahiz bide peza ttipia cen eguin cena, merecimenduzcoa causitcen çuten, herriaren erdira carrosac yuan araztia, aincinian orgac nekes ibilcen ciren lecuyan.

Gauzac certaric hasten diren erraiteco.

Athaicetan bidia hasi eta Administracioniari yin çangon gogora bideco plan berri baten eguiteco [41], Bayonatic Milafranca hartcen dela, ur heguiz, Cambo beheretic ur ona gaindi, Itxassun Garatera, eta Athaizetaric. Pusatuya cen plan hor celhai celacotz eta laburtcen Orçaicen gaindi Donibane Garacira yuaiteco. Gobernamenduya sanyatu cen eta harequin iderac, projeta gueldituya da aintcina seguitu gabe.

Dembora hartan Emperadoria lekhu huntarat yiten celacotz, azqueneco aldian Urriaren 12an 1867 - Athaitzetara, etcen pensatcen baicic agradamenduyen eguitea. Inguignadoreac, M. Bourac, eguinaraci çauncun bidiaren plan bat. Bide haunditic Etchauzan barna, Magnoteneco harroquetarara ; hantic ur heguiz Camboco ur onera. Bidia gostu behar cen 19.000 libera ; bi herriac behar ciren cargatu gastuyez. Conseiluaren deliberacione baten medioz errefusatu çuyen herriac, choilqui planaren eguilia pagatu behar izan cen 56 libera. 
Auzapheça nahi bada ocupatu, hainitz eguin eta [42] eguin araz deçaque herrian. Içaiten dira cuartierrac desiratcen dutenac beren bidiac arranyatcia. Ocasione hortan Auzapheça acort behar da proprietario principalenequin. Eguna hartu lan arte batez malobra borondatezcuena. Hola arranyatu cen Eliza bidetik eta Oxandabaratcia arteco bidia ; billetac emanic Apirilaren 6an 1867an plaçaco cuartierrecueri, Panecau eta Errobico parte bati. Bide gucia dena harri basurec arroilatuya cena, eçarri cen çabalduric eta bardinduric asqui ederqui.

Icusten da bi urthez içan dela Emperadoriaren sokhorria Athaizetaco, Biarritcera yin aldietan, udazquenian. Prefetari emaiten çuyen diruya eta Inguignadoriac partitcen behar cen lekhuetarat. Emaitetic guelditu cen Anqueta bat eguin celacotz Francia gucian industria eta laborançaren gainian. Hori cela medio facilitatu cituyen sokhorriac herrier Prestamuyaren medioz çantima aditionalac emanic edo laugarren malobraren medioz, aintcinago explicacioneac [43] ikhusico diren bezala.

Erretabla Departamenduyan escatcia debecatuya dela eçarri cen. Orobat enseigna bide handian. Athaicetara distancia cembat den indicatcen duyena.

\section{Faceriac}

Faceriac Baztango bailliarequin erreberritu diren aldietan, condicioniac eman dira 1820an eguinetan, zoinaren copia herrian baita. Baztango ardiac hemen libro Mundarrenaraino alhatcera, Itxasucoac han distancia berian iguzquiz iguzqui alha eta bil bacotchac bere lekhuetarat. Behiac eta behorrac libro bordatic athera eta gabaz ala egunaz bere guisa ibilcera.

Guc eguin aintcineco faceriac ciren 1847an, hamar urtheren condicione beretan. Bainan etciren hala pasatu. Biltzarrian Espagnol norbeitec erran behar izan zuyen hitcez arçainic ez libro cedarrietaric aintzina seguitcera ardiac. Hemengo Auçapeçac hala sinhetxi eta etcen curios izan edo ohartu solament [44] faceriac nola eguinac ekhartcen cituyen iracurrarazteco. Cer inteligentcia! Comuzqui horiec dute guehienic desiratzen Auçapez içaitia. Yartcen dira Secretariuac cerbitzatuco dituelacuan. Secretariuac yuanen ditu erresistruac eta correspondantcia, bainan herria obra guti eguinaracico, yeneralian, ez delacotz haren debuarra.

Hemengo arzainac ardien seguitcea ez libro izaite horrec hainitz penaraci cituyen. Guc ere bortz urtheren eguin guinintuyen, çoinac finitu baitciren Urriaren 17an 1865an. Faceriac eguin eta Baztango Alcatia fite sanyatu cen. Sarthu cen berriac nahi izan çuyen faceriac erreberritcia erran nahi baita, arçainac cedarrietaraino ardiac segui eta han abandona desiratcen çuyen. Letrac eguin guintuyen elgarri eta azqueneco asqui seriosac. Beren nahicaria etçuten pasatu. Dembora finitu cenian faceriena, nahi izan guinuyen erreberritcia leheneco condicionetan, errefusatu çuten. Nahi çuten faceriac [45] eguitia bainan ez libro arçainec cedarrietaric aintcinago ardien seguitcera. Pasatu cen dembora aphur bat. Artetan eguiten zauzcuten arrangurac letraz. Hemengo acindac baçabilçala hango lurrian. Azquenecotz bahitcen hasi ciren. Guc ere eman guinintuen ordriac bahitceco. Hortaric lekhora makhurceco arrazoinac emaiten zauzcuten Espagnol arçainec. 
Cedarriac eçarriz bertcen artian, berec nahi çuten lecuyetan. Azquenian peçoina eguin çuten iguzcaico menditic eta $77 \mathrm{co}$ cedarrira heldu artino, Franciacuan sarthuz, erran cedarri hortara heldu baino lehen. Erreclamatu guinen eta conbenitu bidalciaz zoin gure trenchorac fronterala, çoinac bildu baitciren Mayatçaren 23an 1868an. Etciren acomodatu Espagnol Delegatuyac arrazoinatcen çuyen lurren ichuriac eta lephuac heyec gozatu izan diuztela eta usariuac etciuztela galcerat utcico. Prefetari erreporta bat eguin guinuen. Hunec lecuyaren gaineco plan bat eguin guineçala eta bidali izan çancon. Orduyan baciren bi [46] erresumetaco Delegatuyac ocupatcen cirenac fronteraz. Prefetaren desmartchen medioz gure aferaren chuchentceco icendatu ciren Delegatuyac ; hemengo cen M.Boura, Inguignadoria. Afera hoir hola gerthatu cen beçain fite Espainiaco erreguina Isabelle khendu çuten tronutic. Imperiua ere fite erori. Horiec direla medio gueldituya da diferentcia arranyatu gabe. Hain gogor zaudenac, Baztango arthaldedunac faceriac ez eguin nahiz, azquenecotz facerien eguitia ekharraraci ciuzten bertciac arrazoinera. Eta orai hitcez combenituric badabiltza ardiac comunetan çainic gabe.

Huna cembat bahitu ditugun Espagnol hacindac lau urthe eta erdiz ; hasiric Mayatçaren 9an 1866etic eta hacilaren 6an 1870 artino : behorrac 95, behiac 92, astuac 5, ardiac 937, behiac eta behorrac pagarazi 40 sos bakharra, hec hala hasi cirelacotz, eta ardiac bortz sos bakharra. Espagnolec ez dute bahitu guc eguin dugun nombriaren erdia. Gure bahicuntzec munta [47] dute 598 libera 25 centima. Halere ez da gastuyenic bildu ; bethi continuan çain bat fronteran delacotz.

Itxasuco Populuya

Atheratuya Mayatçaren 29an 1866an

\begin{tabular}{|l|l|l|l|}
\hline Cartierrac & Etchiac & Familiac & Yendiac \\
\hline Plaça & 57 & 86 & 245 \\
\hline Panecau & 16 & 22 & 88 \\
\hline Basseburu & 16 & 32 & 107 \\
\hline Erroby & 59 & 96 & 320 \\
\hline Içoquy & 11 & 11 & 51 \\
\hline Guibelarte & 48 & 68 & 262 \\
\hline Ortcia & 15 & 20 & 90 \\
\hline Guerasto & 14 & 15 & 79 \\
\hline Berandotz & 17 & 17 & 65 \\
\hline Olhassour & 27 & 32 & 127 \\
\hline & 280 & 399 & 1434 \\
\hline
\end{tabular}


[48]

Guiçonac :

Ezcongayac

404

Ezconduac

229

Alhargunac

Emaztequiac :

$\begin{array}{ll}\text { Ezcongayac } & 477 \\ \text { Ezconduac } & 232 \\ \text { Alhargunac } & 55 \\ & 764\end{array}$

Presunen totala

\section{Liburuyac}

Gobernamenduya guisa gucietara aplicatcen cen dembora hartan. Içan cen bolta bat nun hiri eta hainitz herritan bibliotequen fondatcia cherkhatcen baizuten. Huna cer guisetara, Ministruec curayestaraciz fundatciaren gainian, aguinduz Gobernamenduyac eguiten tuela laguntzac, herriec emaiten duten arabera. Herri hau ere behar cela figuratu bere heinian deliberatu guinuyen dirua bildu behar cela eta bil arabera liburuyac erosico cirela.

Huna cer publicaraci guinuyen Eliza borthan Abendoaren 15an 1867an.

"Laborantzaren eta bertce hainitz descubrimendu [49] eguin den gaucen gainean, Ministruac desiratcen du yendia yaquinsun eguin dadin. Hortaco nahi du bibliotecac forma diten herri gucietan, ez choilqui haurren escolatceco, bainan ere etchetan dauden yendiac. Cer ere obra desiratcen baita iracurtcia, prestatuco dira, funditu gabe ibilceco condicionerequin. Herri hau ez baita berceac baino guibelatuyago izan behar, suscripcione bat eguinen da particular gucien artian."

Esquiaren medioz bildu cen 82 libera. Hautatu ciren liburuyac eta erosi Parisen, obra ttipiac 35 toma, laborantzaren eta piaya instruicorrez (sic) mintzo direnac. 


\section{Cedarriac}

Comunetaco lurrez herria baliatzen ez delacotz, beriac dituyen bezala, limitac nun tuyen yaquiteco, emanaraci ciren cedarriac proprietariuen eta comunen artian, intresatuyen presentcian, Aphirilaren 23an 24an eta 25an, 1868an, hasiric Guerastotic, Orcia, Cherrenda eta Guibelarten, Bidarraico limitera heldu artino. Cedarrien berificaçailea cen M. Hiribarren [50] Arpenteur géomètre. Aintzinetic hartu çuyen limitetaco copia herrico planetic. Leheneco limitac eta hetan cedarria harrapatu cituyen; escas ciren lekhuetan eçarri ciren. Cedarrien berificacionia eguin cen guero salceco iderarequin. Dembora hartan eta oraino lehenago, Gobernamenduyac nahi çuyen eta engaiarazten cituyen herriac, lur probetchuric emaiten ez duten gucien salcera guero profituya eman deçaqueten guisan. Herri huntan comunen examinatcen ibili cen çuyela bi urthe Conduteur (sic) bat Ponts et Chaussées tacua. Cargua çuyen examinatceco cer clasetaco lurrac ciren. Lurren parte bat arbola landatceco guisa cela eta bertzia acinda hazteco eguin çuyen arraporta. Cedarriac eçarri eta salcerat eman gabe guelditu ciren, bertce aferac presentatu cirelacotz eguin beharrac.

Yendeac yaquin deçan nola ocupatcen diren presuna batçu, huna seguidan cer publicatu guinuyen Eliça borthan hacirilaren 2an 1868an.

[51] "Auçapeçac abisatcen tu comunetan cedarri ateratcen haiçan direnac eçar ditzaten bere lekhuyetan. Bai ora eta berdin gure ondotic yinen direnec, harrapatuco diuztela limitac eta cedarriac, calastraco copia eta plana herrian direno."

\section{Prestamuya}

Bidiac guintuyen hasiac eta icusten lana guelditcera yohan cela erresurxic ez izanez aintcina seguitceco. Gobernamenduyac eman çuyen legue bat çointan facilitatzen baitçuen herrier diruya, prestamuyaren medioz. Herri hau ere baliatu behar cela ocasioneaz, deliberatu guinuyen bilaraztia conseiluya eta guehien contribucionia pagatcen çutenetaric; huna cer erran çancon bilkhuyari, hacirilaren 2an 1868an:

"yuan den Uztailaren 11an eman du Gobernamenduyac leguia Francia gucian bide bicinalen eguiteco. Erresurxac emaiten ditu nahiarequin herriac balia diten. Prestatcen du diruya ehuneco 4an [52] intresa, condicionerequin 30 urthez intresa pagatuco den. Urthe horien buruyan capitala barcatcen du eta bertzalde emaiten prestamuz intresian atheratzen den becembat. Huna comparacionia : mila libera intres eguinez 25.000 libera içanen dira eta bertce hainbertce sokhorri, erran nahi baita 50.000 libera. Diru hori emplegatu behar da hamar urthez bide eguiten. Sokhorri hori emaiten du Gobernamenduyac eçaguturic oraigo sistema seguituz ez ditezquela bidiac eguin. Ministruac Prefetari eman duyen ordriaren arabera yuan den Buruilaren 22an, herriec ez dute dretchoric içanen sokhorri hortan nun ez duten ahalaren arabera enfortic eguiten. 
Gobernamenduyac eçagutu du Anquetaren medioz Francia gucico populuyaren nesesitateric handienea, eta eguin deçaquen fagorerik hoberena dela biden eguiteco sokhorriaren emaitia. Hortacotz bil araci ciuztegu hunara. Intres yeneralaz denian questione seriosqui behar da penxatu. Ororen artian cerbeit eguin behar [53] dugu gure eta ondocuen probetchutan.

Huna cer proposatcen dugun: hirur çantima adicional emendatcia contribucionetan eta ardien taxatcia communen goçatce aldera."

Hasi ciren bacotcha bere opinioniaren elgarri erraiten. Acort ciren azquenian, maiz herrietan guerthatcen dena ez emaitia eta ez eguitiaren gainian. Examinatu ondoan Auzapeçac proposatu çuyena, mayoritatatia nagusitu cen presamuyaren ez eguiteco.

\section{Ardien taxa}

Gobernamenduac herriari socorria emaiteco leguia eman çuenian bide bicinalen eguiteco, orotan clasaraci cituyen, nesesario cirenac eta herriac eguin ala citçazquenac. Herri huntan clasatu guinituyen, lehen clasan. Bide hainditic eta athaicetan gaindi, Bidarraicua heldu artino, orobat lehen clasean, Perusquienetic Oliosenia barna, Otxandabaratcera, eta handi Ordoquira; bigarren clasan, Larrenartetic Haidarrenia barna Espeletarat; hirugarren clasan Indianonetik [54] Fagolara. Ocasionia cen aldi guciez erraiten nioyen Conseiluyari behar guinela baliatu Gobernamenduyari biden eguiteco emaiten duyen sokhorriaz. Erraiten ere çangoten hainitz herritan cer sokhorri içaiten zuten, prestamuya eguin etçutenec ere, laugarren malobraren aldera, edo çantima adicionalac herriari imposaturic. Berri hoyec guciec etcioten batere impresioneric eguiten. Erran nahi çuten bacotcha gure aferac eguin ditçagun eta ez guiten bertcez batere ocupa.

Herriac duyen sokhorria ardien taxatcia cela icusten guinuyen comun goçatce aldera. Zortzi mila ardiren heina hazten da herrico comuneri datchicola. Comunen contribucionia herriac pagatzen duenaz gainean, çucen da taxa paga deçaten acindec. Acindaric ez baladi ibil comunetan, oihanac forma litazke, guisa hortan herriac baluque errebenio frango. Erran diren casu horien gainian, huna cer declaratu çangoten conseilucoeri Oxailaren 7co bilcuyan 1869an :

[55] "Conseilu yeneraleco membruac bildu izan dira yuan den hilabetian Pauben. Gobernamenduyac hamar urthez bidetaco emaiten duyen lehembicico partimenaren eguiteco. 200,000 libera yin dira Departamendu huntara. Sokhorria eman dute cerbeit efort eguin duten herrier, eta Prefetaren arabera hola seguitu gogo dute. Arranguratu dira ceren guelditu guiren guibelerat, hainbertce laguntça içan dugularic eta ontxa abiatu ondoan bide eguiten, yaquiteco dembora hautan cer mobimendu dabilan Francia gucian bide eguiten, gaceten medioz yaquinsun eguin behar liteque. Erranen da nahi dena, holaco mobimenduric ez da icusi eta beldur izaiteco da ez dugun behin icusico bide bicinalen eguitiaren gainian. Herriac pagatcen du comunetaco contribucionia 225 libera urthian. Comun parte bat bederen oihan balitz herriaren sokhorritceco, bainan ez, oro erraiteco maneran buluzac dira. Orai bada Berandotcen arzainen doya, bertce [56] gaineraco lecuyetan ez da betere. Gu herrico conseiluco guiren beçala, herrico intresa guriac baguinitu 
bezala behar ditugu consideratu. Ez deçagun eguin cembeitec nahi luquetena, bercenaren gainian yeneros eta beriac bere daucaten heyec beçala. Yeneros nahi duena içan dadiela beriac dituyen gaucetan. Hainbertce erreparacione bada eguiteco herrian. Eliçatic hasiric etchiac, oihanac formatceco, eta bidiac eguin ahal beçambatian. Ez da herria gobernatu behar etche desordenatu bat beçala, hortaco galdeiten dautzuetegu boçac çucen dena, ardien eta gaineraco bertce animalen taxaceco comunen goçatce aldera."

Hamabi conseilucoetaric çortci present ciren. Bortzec eman çuten boza taxatceco. Bi membruec contra, eta bat guelditu cen bocic eman gabe. Taxa cen hirur sos ardientzat urtheco. Çaldiec eta behiec 20 sos. Içoqui, Errobi eta Basaburucuec bi sos acinda chehiarendaco urthian.

Cer guisaz gueldituyac diren ardiac taxa pagatu [57] gabe erran behar dut, pagatu behar çutela etxia hartuya çutelaric arthaldedunec. Hauyen artian baciren Baquezco yuyiarequin atchiquitcen zutenac. Hari adreçatu ciren pagatu gabe libratceco. Guiçon prudenta izan balitz etcen cargatuco tocatcen ez çancon aferez; yaquin çuyenian deliberacionia hartuya cela ardien tatxaceco, presentatu çauntan ene etchian, erraiteco erretiratu behar nuyela deliberacionia Sous Prefeturatic. Ez nuela erretiratuco erran nioyen, herria bere dretchotan cela. Ez ninçaquela defenda comunetan ibilcetic erran çauntan, usariua celacotz libro içaitia (libre parcours). Erran nioyen herria baçuyela pazcaren pagarazteco dretchua. Despeguitu cen erraiten çaundalaric harc ahalic bazuyen etçutela ardiec pazcaric pagatuco, eta hala guerthatu cen. Dembora hartan Baquezco yuyiac ciren cantoinamenduco administracionearen cerbitçariac, confidenteac. Nola hunec cerbitzu errendatcen baitzuen burupia hartu çuyen ardien taxa ez pagaztetic [58] fagoria behar çuyela galdeguin. Baçaquien dificultatiac emanaracico cituyela. Hasteco Baquesco yuyeak bere meçuz eguinaraci çuyen anqueta bat deliberacionearen contra, non izan baitciren Merian 101 presuna taxaren contra. Arraçoinatu çuten Anquetian bethi danic goçatu ciuztela ardi pazcac pagatu gabe eta hala izaitia pentsatcen çutela.

Seguidan bidali çuyen herrirat Sous-Prefetac deliberacionia, erraiten çuyelaric behar cela publicatu herrian, eta berriz anqueta bat eguin, commodo-incommodo. Lehembicico anquetian bezala batac bercia amoratuz, ardiric etçutenac ere içan ciren bocen emaiten, 103, deliberacionearen contra : bethi arraçoin bera, urrunic goçatu içan tuztela bethi danic ardi pazcac. Nahiz aintcinian ariçan çautan SousPrefetan, M. Cunisse, liburutic articuluyen iracurtcen, eracusterat emaiteco conseiluyac baduela dretchoa ardien taxatceco comunen goçatce alderat, dudaric ez da cinqui (?) adreçatu çancola gure cantoinamenduco Baquesco yuyia [59] M. Castelper; ecic hasi çauntan Sous-Prefeta, correspondenciaz gueroago guehiago ene unharazten, desgustarazten non azquenian herriaren fagoretan obratu behar bidian, lehenbician iduri çuyen beçala, herriaren condenatcen hasi baitcen. Hain cen harrapatuya eta horrequin gogoa histuya, herri huntaco intreseri buruz, non 
azquenian izquiribatu baitçaucun deliberacionea erretiratu behar guinuyela eta publicatu atabala soinuz yendia bilaraciric, erraiteco ardiac ez direla taxatuco herrian. Azquenian horra certara condenatcen guintuyen gure Arrondisamenduco lehen Aintcindariac. Arrapostuya eman çacon, boçac eman behar beitciren Deputatu baten icendatceco, boçac eman artian afera seguitu gabe gueldituco cela. Lagundu behar bidian contrariatuyac baguinen, certaco segui afera?

Administracionian orduco emplegatuac cireno, debalde cen ardiac taxatu nahi izaitia. Hala consideraturic, ez nahiz odol gaichtoric eguin, utcia da projeta gure ondotic yinen direnec executatceco.

\section{[60] Laxeco errienta}

Industria eta laborantça beçala instructionia ere nahi çuyen hedatcia Gobernamenduyac, herri gucietan azquen cuartierreraino. Informacioniac galdeguin cituyen herrietan, heya cuartierrac hedatuyac causitcen ciren. Herri hau araberaca hala consideratu cen. Galdia eguin cen errient batena, deliberacione baten medioz, Oxailaren 16an 1870an , çointan erraiten baitcen Laxeco cuartierrian Errienta nesesario cela, urrundic yin behar cirelacotz haurrac behereco escolara. Berriz ere iduri ez fidaz herriaren engayamenduari, Prefetac har araci çuyen deliberacionia Mayatçaren 20an. Huntan erran çancon, escola emaiteco etchia izaiten cela comoditate onian, bainan herriac bakharric eguin ceçaquen enforta cela mahainen eta alkhien fornitcia escolaco. Ondoco urthian bidali çuten Errienta Laxerat. Bethi danic içanda errientaren plaça eguiten çuyena Laxeco cuartierrian, choilqui iracurtcen eta othoiçac eracusteco heinecua.

\section{[61] Malobretaco laugarren eguna}

Conseiluco membruec nahiz ikhusten çuten bidiac gueldituyac cirela eguitetic, batere etciren embraçatuyac eguin arazteco manerac hartu beharrez; bainan ni auçaphez guisa, eta nahiz hasi ciren lanac segui citen, ez nintuyen uzten ocasionia cen aldi guciez, aiphatu gabe, holaco comoditatea izan eta, ez baliatu nahi içaiteaz Gobernamenduyac biden eguiteco emaiten duyen lauguntzaz. Nahi cen beçanbat aditcerat emanic ere, orobat cen : etçuten sinhesten Gobernamenduyac hainbertce ongui eguin ceçaquela. Ignorantciac berequin dailcana da sinhetx gaitz içaitia. Cinez çabilan Gobernamenduya.

Enperadoriac, Marquis de la Valette Ministruari, erran cioyen camp de Chalossetic, Aboztuaren 15an 1867, anqueta eguin cenaren medioz, yaquina cela yendia cibiliçatuyago cela bidiac eguinac ciren lekhuyetan. 
Ordria eman çuyen Ministruari forma ceçala projet bat cambaretan presentatceco. Projetaren fundamenta behar cela hartu conseilu municipalen deliberacionetaric [62] bide bicinalen eguiteco hamar urthez herriec emaiten duten arabera sokhorrituyac izaiteco. Herri hunec bi urthe pasatcera utci cituyen profitatu gabe.

Azquenian errepetatuyaren medioz, pasaraci ahal içan cen 1870an Oxailaren 6an, condicionerequin emplegatuco cen laugarren eguna Espeletaco bidian, Larrenartetic Haiderrenia barna 7garren eta 8garren numeruan. Nesesario da erraitia deliberacione horren gainian cer guerthatu cen, importantcia handiena çuelacotz erran deliberacioniac çointaric dependitcen baitcen athaicetaco bidiaren eguitia edo ez eguitia.

Deliberacionia hartu eta biharamunian yiten çaut ene etchera conseiluco membro bat, Teillery Martin, ene ondotik Auçaphez yarri dena, erraiteco laugarren eguna conseiluyac imposatcia, herria handia dela, ez dutela ontxa hartuco yendec, behar litezquela bilaraci cembeit laborari, hauyen opinioniaren yaquiteco. Arrapostuya eman nion, conseiluyac baduela [63] facultatia taxatceco, ez nuyela guisa causitcen bi aldiz deliberacionia hartcia, bainan berriz bilceco plazan, lehenbicico igandian publicatuco nuyela Eliza borthan populuyari certaco eman dugun laugarren malobra. Dembora berian Teilleric enseyuac eguin cituyen conseilucoetan deliberacionia hauxaraci nahiz, caria hartara sinatu gabe utcia da deliberacionia, bainan urthe berian Mayatcian, bujeta erreglatu cenian ondoco urtheco, oroc sinatu çuten eta onhartu laugarren eguna. Aintcinian complasent ciren beçala, hasiac ciren contrariatcen conseilucoetan cembait, nola eçagutuco baita Eliza borthan yenderi ene declaratceco maneran, Oxailaren 13an 1870an:

"Yuan den igandian erran den bezala, conseiluyac deliberatu du egun bat urthian emendatcia malobrac, Gobernamendutic içaiteco biden eguiteco sokhorria, bertce herrietan bezala, eta huna cer cantitate içan duten cembeit herrietan, eguin [64] tuzten enforten arabera. Camboco herriac eman ditu hirur çantima adicionel contribucionetan, socorria içan dute Gobernamendutic 600 libera. Bescoitceco herriac laugarren malobraren aldera 933 libera, Ayharren (sic) laugarren malobraren aldera 1428 libera 42, Bastidan orobat 117,81, Bergouin 1117,22. Herri huntan yuyatcen dugu içanen dugula bercetaco arabera mila liberetaric goiti. Bi urthe galtcerat utci ditugu. Orai çortci urthe diru Gobernamendutic socorria içan deçaqueguna. Balia guiten, orai artian eçagutu diren Gobernamenduec ez dute holaco abantailic eman herrien fagoretan.

Nahiz sensu oneco yendia guehiago den herri huntan, badire becaitz direnac, yelos, çazpi edo zortci urthe huntan eguin diren erreparu eta obrez. Ez daquigu arranguratcen diren horiec capable litezquen herrico aferen guidatceco, particularrian berenac eguiten dituzten bezala.

[65] "Satisfamenduya emaiteco orori Gobernamendutic sei urthe huntan içan dugun diruya bide eguiteco pasatcen da hamabi mila liberetan. Dembora berian 
souscripcionez herrian bildu da zazpi mila eta bortz ehun liberaren heina. Diru horietaz icusten duçue cer eguin den herrian. Hasteco Eliza arras yana cen, dorria ustelduya, çur berria eçar araciric ardoasaz estali içan da. Galeriac lanyer handian cirenac, coloma berria eçarri dira quenduric lehenco mendriac. Calostrac oro alchaturic erreparatu dira. Eliça ceruya moldatu eta berritan thindatu. Erretorac M. Salaberric dembora berian aldaren aintcina doraraci eta thindaraci du, bere guisa diruya bilduric herrian. Hilerrietaco portaliac idequiac cirenac burdin grilez eguin dira. Eliçaco cutchac eguin araci oro pheça batian. Ezpeletarat yuaiteco bidia eguin da Basaburungaindi. Bide handitic $n^{\circ} 19$ garrenetic eta athaiz erditaraino carrosac ibil ditazquen bidia eguina da. Herrico mindeya haitz landarez eta gaztenaz [66] ontxa garnituric eçarria da. Erran diren lan horiec guciac eguin dira herrico bujeta behartu gabe, elgar socorrituz behar orduetan bai diruz eta lanez. Gobernatciaren gainian oposicioneric eguin nahi duyenic balinbada eman ditçatela moyen hobiac. Arrangurac bai, bainan moyan hobiac emaitia dudatceco da."

Explicacione hori populuyari eman eta yeneralian satisfos guelditu ciren. Icusten eta eçagutcen zuten behinere harri bat higuitu etcen lecuyan, hainitz gauça eguinac. Lekhu gucietan guertatcen baitira bekhaiztiac heyec eracusterat emaiten çuten pasioniac hartcen cituenian, eguinia ikhusten çuten obra ere, ezdeus baten pare figuraraci nahiz.

\section{Budjeta 1870an}

Haimbertce erreparacione eta bide eguin onduan, hamar urtheric barnian, comparatuz herri huntaco errebenuiequin, oraino guelditcen cen, Mayatcian, ondo urtheco proposatu cen conduen [67] arabera, herriaren disposicioneco 974,11 çantima.

\section{Bozak}

Gobernamenduyac eman çuyen ordria Uztailian 1870an herrico conseiluyac behar cirela berritu. Inperioaren demboran Auzapezac icendatcen ciren orobat conseilutic campo cirenetaric, nola hala beinintçan eguina. Sistema khambiatu cen eta behar cen Préfetac icendatuya conseilucoetaric. Orai errepublicaco leguian herrico conseilucoec behar dute icendatu membro bat beretaric, guisa berian Adjuanta.

Yaquin çutenian arthaldedunec boçac behar cirela, beguiac arguitcen hasi çazcoten, hainbertcetaraino non complota formatu baitcen oposicionia eguin behar çutela conseilu berri baten eguiteco. Motibua çuten ceren cedarriac eçarri ciren comuneri eta particularren arteco muguetan. Gaicituyac, ceren goçatcen diuzten bereri dauden inguruetaco lurrac. Bertçalde ardiac taxatu nahi içaitia [68] comunen goçatce aldera. Beldurrarequin pagaracico çaizcotela arthaldiac ciuztenac elgarri eman ciren. Baciuzten curayastaçaliac : aintcinean izana cen herrian botzqueta 
Deputatu behar cen bat ; erran cioten ematzue boçac enetçat eta ez duçuye ardi pazcaric pagatuco. Halaco aguinçaric eguin gabe bozac bardin izanen cituyen, bainan cer yohan çacote yaun horier herri bat sacrificaturic ere, bere fantesiac pasatcen ahal badituzte. Guisa hortan aguintza horren medioz, oposicionecuac aisiago muntatu ciren, beroquiago elgarri eman. Icusiric contrarietate horiec, utci ciren uhalde batec phasabidia hausterat porchatcen duyen beçala.

Abisatu guinuyen populuya bocen emaitera yiteco, Aboztuaren 7an 1870an. Aincineco beren enforten gainera yendia boz emaitera heldu ceno, etciren baratu conseilu berria nahi çuten partecuac, errecomendacionia eguitetic bozac emaitiaz heyen partecuer. Boçac bildu eta notac hartu ciren. Bederatci botz [69] izan zuyen conseilu berriac guehiago. Hortaric fite conseilu çaharra sanyatu gabe, Gobernamenduyac eman çuyen ordria, ez daquigu arrazoina, berriz behar cirela boçac eman, anulaturic azquenecuac, nahiz contra ordria yin cen ez eguiteco. Arte hortan eguin cen bilçarre bat herrian, berriz icendatceco nor behar ciren proposatu conseiluco. Ni barne cen projeta. Errefusatu nuyen listan ekhartcia, consideraturic asqui dembora cerbitzatu nuela ordu artian herria.

\section{Conseilu berriaren lehen bilzarria.}

Gobernamenduyaren ordrez conseilu berria bildu cen Hacirilaren 1870an. Nahiz Teillery eta Larronde hogoi urthe hartan conseiluco cerbitçatcen ciren herrian, yaquitatez oro bardinsu ciren. Behin ere deus iracurtu eçutenac, administracioneco correspondancia herrico aferen gainian.

Lehembicico gogoratu çangoten afera cen [70] ocupatciaz laur garren malobraz. Oro acort yin ciren, nic emana cela nic behar nuela khen araci. Deliberatu çuten comisionatu bat behar çutela ene gana bidali, eta icendatu çuten conseiluco bat. Presentatu çauntan ene etchian erraiten çautalaric conseiluyaren phartez heldu cela niri erraitera laugarren malobra nic emana dela eta ez badut khentceco moyanac hartcen, deliberacionia hartu gogo duyela conseiluyac, nic imposatia dutala herria egun batez. Iduritcen çangoten ni hola atacatuz debuar handi bat eguiten çutela beren lehen bilçarrian, herriari buruz, popularitatiaren harrapatceco.

Laugarren malobraco egunaren gainian, erran nion mandatariari urthe hartaco emana cela, bainan ondoco urtheco khendu nahi baçuten asqui çutela ez eçartcia bujeta eguitian, nihorc ez cituyela obligaracico eçartcera. Guisa hortan eguin çuten ; etcen baratu Auzapeza [71] bere hasteco opinionia complitu artino; erran nahi baita laugarren malobraren khencia. Cer guiçonaren progreco thalenduya ? Laugarren egun malobretacua emana baitcen numero zazpico eta çortcico bidian emplegatceco, desclasatu ciuzten, iguriquitu gabe cer socorri içanen cen Gobernamendutic. Cer guerthatcen da? 
Bujeta eguin gabe Mayatcian 1872 yiten socorria eta uste baino suma handiagoa Orducotz khendua laugarren eguna, berriz eçartceco lanac ciren ; yenden erranquiçunac batetic khenciaz, bertcetic ceren eman den embraçutan ciren. Halere suprenituyac haimbertce diru yitiaz herrirat, berriz deliberatu çuyen conseiluyac laugarren malobraren emaitera $1873 \mathrm{co}$. Etcen errechqui pasatu alde eta contra bardin izan ciren, Presidentac aurdiqui çuyen balantça.

Seguidan eçarrico dut cembat eman duyen Gobernamenduyac, laugarren malobraren aldera [72] eta cembat urthez.

\begin{tabular}{|l|l|}
\hline 1872an izan cen Gobernamenduyaren socorria & 1440,19 \\
\hline 1873an izan cen orobat Gobernamendutic & 1357,77 \\
\hline Id. Gobernamendutic & 687,51 \\
\hline 1874an izan cen Gobernamendutic & 1433 \\
\hline Id. Departamenducoa & 532 \\
\hline 1875an Gobernamendutic & 661 \\
\hline Id. Departamendutic & 280 \\
\hline 1876an Gobernamenduco socorria Mayatcian & 2194 \\
\hline Id. Id. Ereaurian & 983 \\
\hline Herrian esquia eguinic bilduya & 1440 \\
\hline Totala & 11008,47 \\
\hline
\end{tabular}

Azquenian esquia eguin cen herrian, hurbil idequia celacotz, hasi obra fini çadin amoreagatic. Inguignadoreac M Mugic esperantça emaiten çuyen ardietxico çuyela Gobernamendutic errecompensa, eta hala guerthatu cen. Laxe beherian eguin cen çubia, bideco izan cien dirutic, aurdiquiric 15 metra [73] goraco cen zubi zaharra, harriz eguina, bainan sobera herxia.

Yaun Prefetac quenaraci izan du 1876an, laugarren eguna malobretacoa, erraiten duyelaric leguearen contra emana cela, nahiz dembora hortara artian disimulatu den. Legueac emaiten du lehenic çantima adicionalez behar dela herria imposatu. Ez bada erresurxic asqui çantimequin biden eguiteco, guero aplicatcen da laugarren eguna.

Conseiluya eta guehien imposatuyenetaric cembeit bildu içan dira deliberatceco laugarren egunaren plaçan hirur çantima adicionalez herria cargatceco. Oro acort içan dire ez emaitearen gainean. Guisa hortan icusia da herriac ez duyela guehiago socorriric izanen.

Malurus da herrian energiaric ez duyen conseilu bat izaitia. Hola direnian content içaiten dira sino bat eguin eta iduri cerbeit [74] eguin nahi, guero ez deusetan guelditceco. Lehenago Gobernamenduyec etçuten permititcen ostalera izan çadin Auçapez. Orai leguiac emaiten baitu conseiluyac icendatceco conseilucoetaric, oraicoa hemen hala guerthatcen da. Maiz ostalera içanen da herrian complesent, bere ona hortaric heldu çacolacotz. 
Biden eguiteco socorria izan ceçaquien herriac 1884 artino, emaiteco therminua luçatu çuyelacotz Gobernamenduyac 1872an, erran nahi baita sei urthez eman behar çuyena, hamabiz eman çuyela yin cen ordria.

Eracusterat emaiteco nic eguin auçapezguaren memoriaren copia dela, sinatcen dut Buruilaren hogoi ta lauan, mila zortci ehun eta hirur eta hogoi eta hemezortcian.

\section{Guillaume Berrouet}

Itsasssun sortuyan Etchedoyan (Ereauriaren) hamabortcian, mila zortci ehun eta hamaseyan. Aita Etienne Berrouet edo Berruet eta Ama Maria Bergez.

G. B.

Déposé à la mairie d'Itxassou pour faire partie des archives suivant le désir que M. Berrouet Guillaume a exprimé avant de mourir.

Itxassou, le 8 octobre 1890.

Le Maire,

D. Berrouet 